\title{
REVIEWS
}

Check for updates

\section{The germanium quantum information route}

\author{
Giordano Scappucci(i] ${ }^{\boxplus 凶}$, Christoph Kloeffel ${ }^{2}$, Floris A. Zwanenburg ${ }^{3}$, Daniel Loss $\mathbb{D}^{2}$, \\ Maksym Myronov $\mathbb{D}^{4}$, Jian-Jun Zhang ${ }^{5}$, Silvano De Franceschi ${ }^{6}{ }^{6}$, Georgios Katsaros ${ }^{7}$ \\ and Menno Veldhorst (iD)
}

Abstract | In the effort to develop disruptive quantum technologies, germanium is emerging as a versatile material to realize devices capable of encoding, processing and transmitting quantum information. These devices leverage the special properties of holes in germanium, such as their inherently strong spin-orbit coupling and their ability to host superconducting pairing correlations. In this Review, we start by introducing the physics of holes in low-dimensional germanium structures, providing key insights from a theoretical perspective. We then examine the materials-science progress underpinning germanium-based planar heterostructures and nanowires. We go on to review the most significant experimental results demonstrating key building blocks for quantum technology, such as an electrically driven universal quantum gate set with spin qubits in quantum dots and superconductor-semiconductor devices for hybrid quantum systems. We conclude by identifying the most promising avenues towards scalable quantum information processing in germanium-based systems.

'QuTech and Kavli Institute of Nanoscience, Delft University of Technology, Delft, Netherlands.

${ }^{2}$ Department of Physics, University of Basel, Basel, Switzerland.

${ }^{3} \mathrm{MESA}+$ Institute for Nanotechnology, University of Twente, Enschede, Netherlands.

${ }^{4}$ Department of Physics, University of Warwick, Coventry, UK.

${ }^{5}$ Institute of Physics and CAS Center for Excellence in Topological Quantum Computation, Chinese Academy of Sciences, Beijing, China.

${ }^{6}$ Univ. Grenoble Alpes and CEA, IRIG/PHELIQS Grenoble, France.

${ }^{7}$ Institute of Science and Technology Austria, Klosterneuburg, Austria.

凶e-mail:g.scappucci@ tudelft.nl

https://doi.org/10.1038/ \$41578-020-00262-z
The first quantum revolution has led to a microscopic understanding of nature, enabling the development of transistors. These were first made of germanium, owing to its appealing electrical properties. Silicon took over as the material of choice for microelectronics because of the exceptional quality of silicon dioxide, allowing for the fabrication and integration of increasingly smaller transistors. Two key breakthroughs are now fuelling a renaissance of Ge-based materials and technologies ${ }^{1,2}$. First, the maturity achieved by $\mathrm{Ge}$-compatible dielectrics with high dielectric constant $(\kappa)$ overcomes the lack of a stable native oxide; second, the heterogeneous integration of $\mathrm{Ge}$ on $\mathrm{Si}$ within a conventional complementary metal-oxidesemiconductor process bypasses the need for developing $\mathrm{Ge}$ substrates at prohibitive manufacturing costs. Germanium has the highest hole mobility of semiconductors at room temperature and, therefore, is considered a key material in a 'More than Moore' approach to extend chip performance in classical computers beyond the limits imposed by miniaturization.

Because of the unique combination of intrinsic materials properties and compatibility with existing complementary metal-oxide-semiconductor technology, $\mathrm{Ge}$ is also emerging as a promising material within the current second quantum revolution, in which quantum matter is engineered to develop disruptive technologies beyond the reach of classical understanding. Quantum confined holes in $\mathrm{Ge}$ are a compelling platform for quantum technologies based on spins ${ }^{3}$, topological states ${ }^{4}$ and gate-controlled superconducting qubits (gatemons) ${ }^{5}$.

Its high hole mobilities benchmark $\mathrm{Ge}$ as an ultraclean material platform for well-controlled, high-quality quantum dots (QDs). The low effective mass of charge carriers in $\mathrm{Ge}$, tunable by confinement and strain, results in QDs with large energy-level spacing, allowing to relax lithographic-fabrication requirements, which is advantageous because uniformity and ease of fabrication are critical for scaling up to large quantum systems. Long hole-spin lifetimes are also important, and, in Ge, they are expected for two reasons: first, the hyperfine interaction is suppressed due to the p-type character of the valence band and, second, Ge can be engineered by isotopic purification into a nuclear spin-free material. Other important properties of holes in Ge are their large and tunable $g$-factors and spin-orbit interaction energies. These are key ingredients to realize fast, all-electrical spin-qubit control without the need for any microscopic structure, for spin-qubit coupling at a distance via superconductors and for the emergence of Majorana zero modes (MZMs) for topological quantum computing.

From a fabrication perspective, virtually every metal on $\mathrm{Ge}$ shows a Fermi level pinned close to the valence band $^{6}$, including superconductors. As a consequence, it is straightforward to make ohmic contact to confined holes in $\mathrm{Ge}$, without the need for local doping 
or implantation with the associated high-temperature annealing step. Furthermore, the low Schottky barrier at the metal-semiconductor interface facilitates the formation of transparent contacts to superconductors, a key element in semiconductor-superconductor hybrids. Most importantly, Ge is a foundry-compatible material, enabling advanced device manufacturing and integration. This is crucial for advancing to large-scale quantum systems, as many challenges related to epitaxy, dielectrics and variations of critical device dimensions may be solved by resorting to the advanced process control in a state-of-the-art manufacturing facility ${ }^{7}$.

These attractive properties have motivated extensive research efforts in the past several years. Advances in the optimization of Ge-based materials and in the understanding of their underlying physics have led to impressive achievements. In this Review, we specifically focus on hole-based devices relevant to the developing field of quantum computation. Three materials platforms have emerged as strong contenders in the race to build quantum information-processing devices in germanium: Ge/Si core/shell nanowires (NWs), Ge hut wires (HWs) and Ge/SiGe planar heterostructures. Each of these platforms offers specific advantages but also poses challenges, as we shall discuss. Recent milestones in Ge quantum technology include a record hole mobility ${ }^{8,9}$ of up to $10^{6} \mathrm{~cm}^{2}(\mathrm{Vs})^{-1}$; the growth of site-controlled $\mathrm{HWs}^{10}$; the fabrication and measurement of QDs in NWs, HWs and quantum wells ${ }^{11-18}$; the demonstration of electrically driven spin qubits ${ }^{15}$, fast two-qubit logic ${ }^{19,20}$ and coherent control, manipulation and readout of single-hole spins $^{21}$; the demonstration of gate-tunable superconductivity, superconductor-semiconductor (S-Sm) hybrids and the coupling of a hole charge to a superconducting resonator $^{17,18,22-24}$.

This Review is organized as follows. We start by introducing the physics of holes in Ge, providing theoretical insights relevant to the use of $\mathrm{Ge}$ as a material for qubits based on spin and topological states. We discuss the materials-science developments in the growth and electrical characterization of $\mathrm{Ge} / \mathrm{SiGe}$ planar heterostructures, Ge/Si core-shell NWs and Ge HWs, the three platforms that have enabled building blocks for Ge quantum technology so far. We then summarize the state of experimental progress in Ge devices for quantum technology, which provide initial confirmations of the theoretical promises. We start with results for QDs, including spin states, $g$-factors, charge noise and Pauli spin blockade. We then discuss hole-spin qubits experiments, including single-shot spin readout, electrically driven spin qubits and fast two-qubit logic. Finally, we cover proximity-induced superconductivity in S-Sm hybrids, specifically, Josephson field-effect transistors (JoFETs), superconducting quantum-interference devices, superconducting quantum point contacts and QDs. Based on theoretical considerations and experimental results, we end by presenting our vision for the development of Ge quantum technology towards, for example, macroscopic entanglement between spins, quantum error-correction architectures and coherent transfer of quantum information between spin and topological qubits.

\section{Physics of holes in germanium}

Only a few years after spins in QDs were proposed for quantum computation ${ }^{3}$, magnetically and electrically driven single-spin rotations and sub-nanosecond $\sqrt{\text { SWAP }}$ gates between two spins were demonstrated with conduction-band electrons in lateral GaAs QDs ${ }^{25-28}$. Around the same time, theoretical studies ${ }^{29-32}$ showed that not only filled conduction-band states but also holes, that is, unfilled valence-band states, are promising candidates for the implementation of spin qubits. The potential of holes for quantum computation was underlined by the encouraging hole-spin lifetimes measured in self-assembled InGaAs QDs ${ }^{33-36}$. By now, holes have been studied in a variety of materials and nanostructures. In this section, we focus on holes in Ge-based devices and provide some key theoretical insights.

\section{Heavy-hole and light-hole states}

Bulk crystal. In the electronic band structure of bulk $\mathrm{Ge}$ (unstrained, diamond cubic lattice), four degenerate valence-band states of highest energy are located at the $\Gamma$ point (FIG. 1a), where the crystal momentum $\hbar \boldsymbol{k}$ of the electrons is zero. However, by combining the degrees of freedom due to the electron spin and those due to the p-type atomic orbitals, a sixfold instead of fourfold degeneracy would be expected. As we explain in the following, the sixfold degeneracy is lifted by a spin-orbit interaction at the atomic level. This interaction separates the four states in which the bare spin and the atomic orbital angular momentum are parallel (effective spin $3 / 2$ ) from the remaining two states, in which they are antiparallel (effective spin 1/2). More precisely, a term of type $\delta_{\mathrm{so}} \boldsymbol{l} \cdot \boldsymbol{s}$ can be derived from the Dirac equation ${ }^{37}$, where $\delta_{\text {so }}$ is a coefficient, $\hbar \boldsymbol{l}$ the angular momentum associated with the p-type atomic orbitals and $\hbar s$ the bare electron spin. Consequently, an effective spin $\hbar \boldsymbol{j}=\hbar(\boldsymbol{l}+\boldsymbol{s})$ can be defined. From the quantum-mechanical addition of angular momenta and the properties $\boldsymbol{l} \cdot \boldsymbol{l}=l(l+1)$ and $\boldsymbol{s} \cdot \boldsymbol{s}=s(s+1)$ with $l=1$ and $s=1 / 2$, it follows that $\delta_{\mathrm{so}} \boldsymbol{l} \cdot \boldsymbol{s}$ has four degenerate eigenstates with $j=3 / 2$ and eigenenergy $\delta_{\mathrm{SO}} / 2$, and two degenerate eigenstates with $j=1 / 2$ and eigenenergy $-\delta_{\mathrm{SO}}$. The introduced quantum number $j$ is related to the size of the effective spin via $\boldsymbol{j} \cdot \boldsymbol{j}=j(j+1)$. The energy difference $\Delta_{0}=3 \delta_{\mathrm{SO}} / 2$ is commonly referred to as the spin-orbit gap and separates the topmost valence band $(j=3 / 2)$ from the spin-orbit split-off band $(j=1 / 2)$ at the $\Gamma$ point $^{38}$. For Ge, $\Delta_{0} \approx 0.3 \mathrm{eV}$ is relatively large, and, so, the valence-band states with effective spins $3 / 2$ and $1 / 2$ are energetically more separated than, for example, in $\mathrm{Si}$, where $\Delta_{0}$ is only about $44 \mathrm{meV}\left(\mathrm{REF}^{38}{ }^{38}\right.$.

Near the $\Gamma$ point, the states in the topmost valence band of Ge are well described by the Luttinger-Kohn Hamiltonian $^{39,40}$ in the spherical approximation ${ }^{41}$ :

$$
H_{\mathrm{LK}}=-\frac{\hbar^{2}}{2 m_{0}}\left[\left(\gamma_{1}+\frac{5}{2} \gamma_{s}\right) k^{2}-2 \gamma_{s}(\boldsymbol{k} \cdot \boldsymbol{J})^{2}\right],
$$

where $k^{2}=\boldsymbol{k} \cdot \boldsymbol{k}=k_{x}^{2}+k_{y}^{2}+k_{z}^{2}, m_{0}$ is the free electron rest mass, $\hbar \boldsymbol{J}$ is the operator for an effective spin $3 / 2$, and $\gamma_{1}$ and $\gamma_{s}$ are material-dependent parameters. The term $\boldsymbol{k} \cdot \boldsymbol{J}$ illustrates a crucial feature of hole states: the crystal momentum and the effective spin are closely linked 

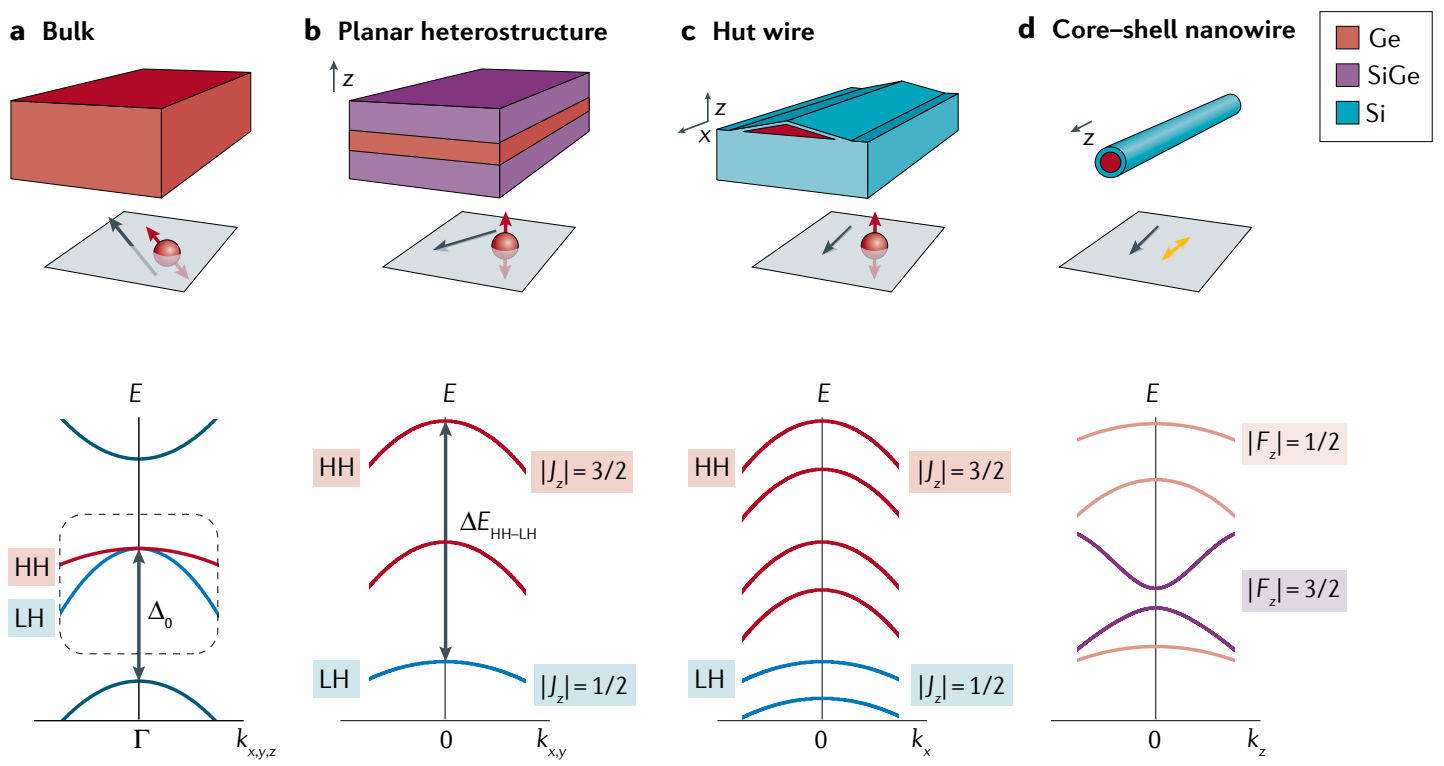

Fig. 1 Quantum confined holes in germanium. a In bulk Ge, electrons and holes can move in all directions. An excerpt from the electronic band structure is sketched at the bottom. The heavy-hole $(\mathrm{HH})$ and light-hole $(\mathrm{LH})$ bands are described by Eq. 1 , with the effective spin and momentum satisfying $\cdot \mathbf{k} / \mathrm{k}= \pm 3 / 2 \mathrm{for} \mathrm{HH}$ (inset). Consequently, the effective spin (red arrow) of a $\mathrm{HH}$ is parallel or antiparallel to its direction of motion (black arrow). Corrections beyond the spherical approximation are sometimes relevant ${ }^{38,41,43,51}$ and not considered here for simplicity. In all remaining panels, we zoom in on spectra originating from the bulk HH and LH bands (dashed box). b $\mid$ Holes in a Ge/SiGe quantum well can propagate along any in-plane direction. Sub-bands of $\mathrm{HH}$ and $\mathrm{LH}$ types arise because of size quantization and strain. Sketched are the two highest $\mathrm{HH}$ sub-bands (red) and the topmost $\mathrm{LH}$ sub-band (blue). The $\mathrm{HH}$-LH splitting is given by $\Delta E_{\mathrm{HH}-\mathrm{H}}$. If corrections are taken into account, the in-plane spectrum becomes anisotropic. The inset illustrates a pure $\mathrm{HH}$ whose effective spin (red arrow) is parallel or antiparallel to the out-of-plane direction. While the direction of motion (black arrow) in panel $\mathbf{b}$ can be any in-plane direction, the holes in panels $\mathbf{c}$ and $\mathbf{d}$ propagate along the wires. $\mathbf{c}$ | For Ge hut wires, the additional hole confinement along one of the in-plane axes results in additional sub-bands compared with panel $\mathbf{b}$, a few of which are drawn. Here and in panel $\mathbf{b}$, small $\mathrm{HH}-\mathrm{LH}$ mixings lead to deviations from $J_{z}= \pm 3 / 2$ (red arrows) and $J_{z}= \pm 1 / 2$ for the sub-bands of $\mathrm{HH}$ and $\mathrm{LH}$ types, respectively. $\mathbf{d} / \operatorname{In} \mathrm{Ge} / \mathrm{Si}$ nanowires, the effective spin states related to $J$ are closely linked to the orbital parts of the envelope wave functions and usually strongly mixed. However, due to an approximately cylindrical symmetry, the sub-bands can be classified according to the total angular momentum $\hbar F_{z}$ along the wire $e^{47,49-51}$. The difference $\hbar\left(F_{z}-J_{z}\right)$ results from orbital angular momenta given by the envelope functions. If cylindrical symmetry is assumed, the topmost sub-bands satisfy $F_{z}= \pm 1 / 2$ (yellow arrow, inset).

to each other. In particular, the eigenstates of $H_{\mathrm{LK}}$ can be grouped into heavy-hole $(\mathrm{HH})$ and light-hole $(\mathrm{LH})$ states. In the case of $\mathrm{HH}$ states, the effective spin is parallel or antiparallel (spin projections of $\pm 3 \hbar / 2$ ) to the hole's direction of motion (given by $\boldsymbol{k}$ ) and the associated eigenenergy is $-\hbar^{2} k^{2} / 2 m_{\mathrm{HH}}$, where $m_{\mathrm{HH}}=m_{0} /\left(\gamma_{1}-2 \gamma_{s}\right)$ is the effective $\mathrm{HH}$ mass. In the case of LH states, the spin projection along the direction of motion is only $\pm \hbar / 2$ and the eigenenergy is $-\hbar^{2} k^{2} / 2 m_{\mathrm{LH}}$, where $m_{\mathrm{LH}}=m_{0} /\left(\gamma_{1}+2 \gamma_{s}\right)$ is the effective LH mass. For Ge, one finds ${ }^{42} \gamma_{1} \approx 13$ and $\gamma_{s} \approx 5$. Consequently, $m_{\mathrm{HH}}$ is about an order of magnitude greater than $m_{\mathrm{LH}}$.

Planar heterostructures. The characteristic couplings between the effective spins and the crystal momenta of holes have important consequences when confinement by an external potential is present. In $\mathrm{Ge} / \mathrm{SiGe}$ planar heterostructures (FIG. 1 b), holes are confined to the strained Ge layer because of the valence-band offsets at the interfaces. Because the hole confinement in the out-of-plane direction $z$ is much stronger than in the $x-y$ plane, these heterostructures can be considered as quasi-2D systems. Given that the variance of a hole's $z$-coordinate is small, Heisenberg's uncertainty principle implies that the variance of the out-of-plane momentum has a large lower bound. As a consequence, basis states that originate from the topmost valence band are usually classified according to the out-of-plane component $\hbar J_{z}$ of the effective spin. More precisely, basis states satisfying $J_{z}= \pm 3 / 2$ and $J_{z}= \pm 1 / 2$ in a quasi-2D structure are commonly referred to as $\mathrm{HH}$ and $\mathrm{LH}$ states, respectively ${ }^{38}$. These are related but not identical to the $\mathrm{HH}$ and $\mathrm{LH}$ states in a bulk semiconductor.

Recent calculations ${ }^{43}$ for realistic Ge/SiGe quantum wells showed that size quantization and strain ${ }^{44}$ induce a large splitting of more than $100 \mathrm{meV}$ between $\mathrm{HH}$ and $\mathrm{LH}$ states, with $\mathrm{HH}$ states energetically favoured. Hence, removing valence-band electrons from quasi-2D Ge systems usually results in hole states whose spin parts consist predominantly of states for which $J_{z}= \pm 3 / 2$. Assuming pure $\mathrm{HHs}^{38,45}$, the $k_{x, y}$-dependent terms of Eq. 1 simplify to $-\hbar^{2}\left(k_{x}^{2}+k_{y}^{2}\right) / 2 m_{\|}^{\mathrm{HH}}$, where $m_{\|}^{\mathrm{HH}}=m_{0} /\left(\gamma_{1}+\gamma_{s}\right) \approx 0.055 m_{0}$ is remarkably light. If, instead, $J_{z}= \pm 1 / 2$ (pure LHs), $m_{\|}^{\mathrm{HH}}$ is replaced by the heavier mass $m_{\|}^{\mathrm{LH}}=m_{0} /\left(\gamma_{1}-\gamma_{s}\right)$, meaning that $\mathrm{HH}$ and LH sub-bands have reversed characters for the in-plane motion $^{38}$. Indeed, calculations ${ }^{43}$ and experiments with strained, undoped $\mathrm{Ge} / \mathrm{SiGe}$ quantum wells ${ }^{46}$ resulted in very light in-plane effective masses at the top of the valence band (low-energy holes) of about $0.05 m_{0}$; the 
exact values depend on details such as the strain. Small effective masses lead to large orbital-level spacings in QDs and are, therefore, useful, for example, for the implementation of spin qubits.

Hut wires and nanowires. Germanium HWs (FIG. 1c) grown on Si have a triangular cross section and are fully compressively strained along the in-plane directions $x$ and $y$, where they adopt the Si lattice constant. Given the large width-to-height aspect ratio of about 10, holes are very strongly confined in the out-of-plane direction $z$. Calculations $\mathrm{s}^{10,14}$ suggest that low-energy holes in $\mathrm{Ge}$ $\mathrm{HWs}$ are nearly pure HHs with a $\mathrm{LH}$ admixture below $1 \%$. Consequently, their effective mass for the motion along the HW is expected to approach the small in-plane mass of low-energy holes in planar heterostructures.

In Ge/Si core-shell NWs (FIG. 1d), the large valenceband offset of about $500 \mathrm{meV}$ at the $\mathrm{Ge} / \mathrm{Si}$ interface confines holes to the Ge core. In contrast to the aforementioned systems, there is more than one axis of strongest hole confinement, typically leading to eigenstates that exhibit large HH-LH mixings, even at low energies ${ }^{47-49}$. The motion of low-energy holes along the NW axis $z$ is well described by the term $\hbar^{2} k_{z}^{2} / 2 m_{\text {eff }}$, where the effective mass $m_{\text {eff }}$ depends strongly on the strain ${ }^{50,51}$. Therefore, $m_{\text {eff }}$ is determined by details of the device, such as the thickness of the Si shell ${ }^{52,53}$. Calculations showed that $m_{\text {eff }}$ can range from small values $\left(<0.1 m_{0}\right)$, appealing for spin qubits ${ }^{54,55}$, to infinity and even change $\operatorname{sign}^{50,51}$. Large effective masses reduce the kinetic-energy term and, thereby, lead to a relative increase of the effects of electron-electron interactions ${ }^{56}$. This has particularly interesting implications for Majorana physics with holes ${ }^{56-58}$.

\section{Spin-orbit interaction and $g$-factors}

The diamond cubic structure has a centre of inversion, which means that $\mathrm{Ge}$ is bulk-inversion-symmetric. By contrast, the material interfaces and the external potential in Ge-based devices can induce inversion asymmetry and, consequently, a spin-orbit interaction at the level of the envelope wave functions ${ }^{38}$. Effects of interface-induced inversion asymmetry on the spinorbit interaction of electrons and holes have been studied in detail for a variety of systems ${ }^{59-69}$.

About ten years ago, it was predicted that holes in $\mathrm{Ge} / \mathrm{Si}$ NWs would feature a strong, direct Rashba spinorbit interaction (DRSOI) ${ }^{50,51}$ under the application of an electric field perpendicular to the wire. The DRSOI requires $\mathrm{HH}-\mathrm{LH}$ mixing and can be switched on and off via gates. In contrast to the well-known case of conduction-band electrons in Rashba NWs, the effective DRSOI coefficient does not depend on couplings between the conduction-band and valence-band states, and can, therefore, be exceptionally large, as demonstrated in experiments $\mathrm{s}^{70-75}$. For example, spin-orbit energies of a few $\mathrm{meV}$ were recently measured for holes in $\mathrm{Ge} / \mathrm{Si} \mathrm{NWs}^{73,74}$. According to theoretical studies, a moderate electric field in the Ge core is sufficient to generate such high spin-orbit energies ${ }^{50,51}$ and, for example, allows for efficient hole-spin qubit rotations ${ }^{54}$ via electric dipole spin resonance ${ }^{76}$.
For systems such as $\mathrm{Ge} / \mathrm{SiGe}$ heterostructures and Ge HWs, in which the hole confinement in one direction clearly exceeds that in the other directions, the DRSOI is less pronounced on account of a large $\mathrm{HH}-$ LH splitting ${ }^{10,51}$. However, it is known from studying conduction-band electrons in lateral QDs ${ }^{77-79}$ that there are many spin-orbit interaction terms that can be of high relevance. For holes in quasi-2D systems, great efforts in both theory and experiment have led to detailed insights into the spin-orbit interaction ${ }^{10,30,38,41,43,80-91}$. Calculations for QDs in planar $\mathrm{Ge} / \mathrm{SiGe}$ heterostructures ${ }^{43}$ predicted that the spin-orbit interaction of hole-spin qubits therein can be harnessed to perform rapid (few nanoseconds) qubit rotations by means of electric dipole spin resonance, as was confirmed by experiments ${ }^{15,19,20}$.

Many applications of Ge-based devices require the presence of a magnetic field $\boldsymbol{B}$, which we now add to the discussion. Remarkably, the effective $g$-factors of holes (related to the effective Zeeman splittings $\left|g \mu_{\mathrm{B}} \boldsymbol{B}\right|$, where $\mu_{\mathrm{B}}$ is the Bohr magneton) depend strongly on the confinement potential and the magnetic-field orientation $^{38,41,45,49,50,84,92-94}$. For Ge HWs, Ge/SiGe structures and similar quasi-2D systems, a pure-HH approximation $^{45}$ suggests that the effective $g$-factors $g_{\perp}$ and $g_{\|}$for an out-of-plane and in-plane $\boldsymbol{B}$, respectively, satisfy $\left|g_{\perp}\right| \gg\left|g_{\|}\right|$, as usually observed in experiments ${ }^{10,14,45,82,95}$. However, a pure-HH approximation is often insufficient. For example, it turns out that even tiny LH admixtures can substantially reduce the out-of-plane $g$-factor ${ }^{13,14,84,96-99}$. Nevertheless, we note that a ratio $\left|g_{\perp} / g_{\|}\right|$of up to 18 was measured for holes in Ge $\mathrm{HWs}^{14}$. A particularly attractive property of holes, which is found in various structures, is a strong dependence of the effective $g$-factor on the applied electric field ${ }^{13,54,72,75,100-103}$. This property can be used, for example, to electrically tune the Zeeman splitting and, hence, the resonance condition of hole-spin qubits ${ }^{21}$. Furthermore, it allows for electrically driven spin rotations via $g$-matrix modulation $^{100,102,104,105}$.

\section{Hole-spin relaxation and decoherence}

A key criterion for high-quality qubits is that the decoherence time should be much longer than the gateoperation times ${ }^{106}$. Unlike III-V materials, Ge and Si contain only a small percentage of atomic nuclei with non-zero spins. Isotopic purification ${ }^{107-113}$ even allows for (almost) nuclear-spin-free devices. The contact hyperfine interaction between the remaining nuclear spins and hole spins is suppressed because the p-type atomic orbitals vanish at the nuclei $i^{31,34,36,114,115}$. It is also worth noting that valley degeneracies, which are present in the lowest conduction bands of $\mathrm{Ge}$ and $\mathrm{Si}$, are absent in their topmost valence bands. Thus, it is highly probable that neither valley degrees of freedom ${ }^{116,117}$ nor nuclear spins are ultimately a limiting factor for hole-spin lifetimes in Ge-based devices.

As a result of the strong spin-orbit interaction and the electric-field-dependent $g$-factors, charge noise is a major source of hole-spin relaxation and decoherence. Fortunately, it is usually possible to identify sweet spots ${ }^{118-124}$ where qubits are, to a large extent, insensitive to electrical noise. For example, a sweet spot for an idle 


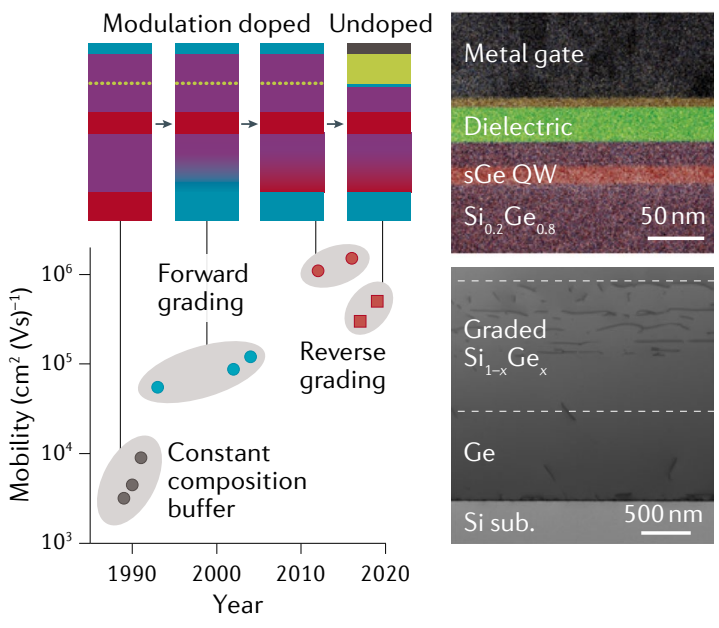

b Fractional quantum Hall effect

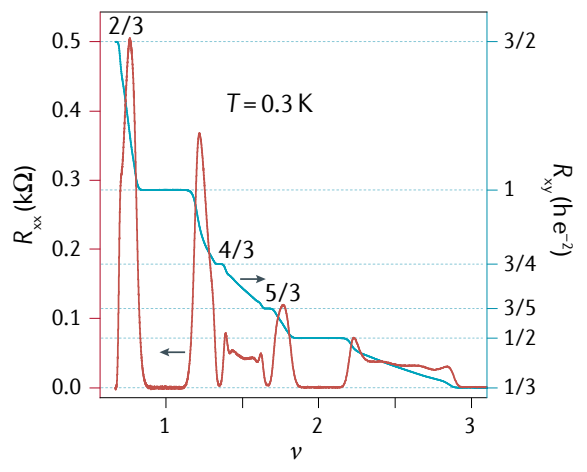

c Ballistic transport

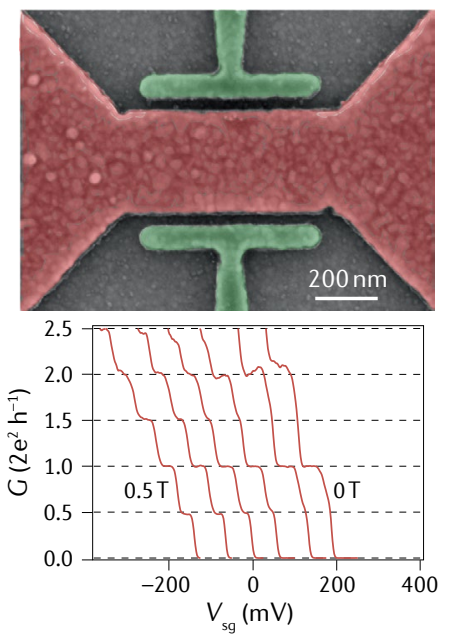

Fig. 2 | Planar Ge/SiGe heterostructures. a Improvements in the low-temperature hole mobility through the years (left panel) obtained thanks to the evolution towards top-gated undoped Ge quantum wells (QWs) with high- $\kappa$ dielectric/ metal gate stack (top-right panel) on reversed-graded relaxed SiGe buffers (bottom-right panel). The image of the reversegraded buffer was obtained by scanning transmission electron microscopy, and that of the QWs with scanning transmission electron microscopy and energy-dispersive X-ray analysis. Mobility data points are extracted from REFS ${ }^{136-168}$ (black circles), REFS ${ }^{139,143,144}$ (blue circles), REFS $^{8,174}$ (red circles) and REFS ${ }^{9,170}$ (red squares). b | Longitudinal resistance, $R_{\mathrm{xx}}$, and Hall resistance, $R_{x y}$ of a $2 \mathrm{D}$ hole gas in Ge/SiGe as a function of filling factors $(v)$, showing Shubnikov-de Haas minima and quantum Hall plateaus at fractional filling factors. c | Scanning electron microscope image of a ballistic 1D channel in planar Ge/SiGe that is defined by a channel gate and a side gate, and that shows quantized conductance, $G$, as a function of side-gate voltage, $V_{\mathrm{sg}}$. Lifting of spin degeneracy is observed at finite magnetic field. Panel $\mathbf{a}$ (right) is adapted with permission from REF. ${ }^{\text {, }}$ Wiley-VCH Verlag GmbH \& Co. Panel $\mathbf{b}$ is adapted with permission from REF. ${ }^{163}$, American Physical Society. Panel $\mathbf{c}$ is adapted with permission from REF. ${ }^{176}$, American Chemical Society.

hole-spin qubit in a Ge/Si NW QD is reached when the effective electric field therein and, hence, the DRSOI of the hole is zero ${ }^{54}$. Thus, by switching the electric field off unless needed for quantum logic gates, the qubit can be very well isolated from its environment. It is worth noting that, even at sweet spots, high-order processes can lead to substantial relaxation and decoherence, and, so, reducing the effects of charge noise as much as possible is an important research topic. Phonons ${ }^{28,78,116,125-128}$ are another considerable noise source. However, piezoelectric hole-phonon coupling is, to a certain extent, suppressed in Ge-based devices because of the bulk inversion symmetry of Si and Ge. Moreover, calculations for hole-spin qubits ${ }^{29,32,101,129}$ showed that the phonon-mediated qubit decay can be substantially slowed down, if necessary. One option, for instance, is to change the strength or orientation of the magnetic field.

\section{Materials platforms}

High-quality materials are crucial for viable quantum experiments and quantum technology. In this section, we describe the developments that have established planar heterostructures, NWs and HWs as the platforms of choice to investigate the properties of holes in Ge that are relevant for quantum information.

\section{Planar heterostructures}

In $\mathrm{Ge} / \mathrm{SiGe}$ planar heterostructures, the band-edge alignment between compressively strained $\mathrm{Ge}$ and relaxed $\mathrm{Si}_{1-x} \mathrm{Ge}_{x}$ produces a type I band alignment that confines holes in the growth direction, creating a $2 \mathrm{D}$ hole gas $(2 \mathrm{DHG})^{130-132}$. Differently than Ge/Si NWs and Ge HWs, $\mathrm{Ge} / \mathrm{SiGe}$ heterostructures may also support confinement of $2 \mathrm{D}$ electron gases, because a sizable conduction-band offset can be achieved under appropriate strain and composition conditions ${ }^{133}$. The compressive strain in the epitaxial Ge quantum well is set by the in-plane lattice parameter of the underlying relaxed $\mathrm{Si}_{1-x} \mathrm{Ge}_{x}$ buffer layer. In practice, these $\mathrm{Si}_{1-x} \mathrm{Ge}_{x}$ buffers require a high $\mathrm{Ge}$ content $(0.6 \leq x \leq 0.9)$ to support strained Ge epilayers of a reasonable thickness $(10-30 \mathrm{~nm})^{134}$, smaller than the critical thickness for the onset of plastic relaxation ${ }^{135}$. The Ge quantum well can be populated with holes from a remote supply layer (modulation doping) or from the ohmic contacts via gate-induced accumulation in undoped heterostructures.

The compressive strain in the Ge quantum well produces large and tunable energy splittings between the $\mathrm{HH}$ and LH bands, suggesting that Ge could host a 2DHG of very high mobility, because the topmost $\mathrm{HH}$ band has a very light effective mass for the in-plane motion, as we discussed in the section on the physics of holes in Ge. Motivated by the pursuit of high-mobility p-type channels, modulation-doped strained $\mathrm{Ge} / \mathrm{SiGe}$ heterostructures were pioneered in the 1990s by using molecular-beam epitaxy. The highest low-temperature hole-mobility values improved from less than $10^{4} \mathrm{~cm}^{2}(\mathrm{Vs})^{-1}$ in constantcomposition SiGe buffers on Ge substrates ${ }^{136-138}$ to $5.5 \times 10^{4} \mathrm{~cm}^{2}(\mathrm{Vs})^{-1}\left(\mathrm{REF}^{139}\right)$ in composition-graded SiGe buffers on Si substrates (FIC. 2a). 
The progress was modest because several microns of epitaxial growth were required to accommodate the large lattice mismatch between Ge-rich SiGe and the underlying Si substrate, producing increased roughness and high threading dislocation density $\left(\geq 10^{7} \mathrm{~cm}^{-2}\right)$ during the long-lasting growth. These early efforts are reviewed in REFS ${ }^{140,141}$. One strategy for improving the growth of Ge-rich SiGe was to introduce a very steep gradient to $\mathrm{Si}_{0.3} \mathrm{Ge}_{0.7}$ layers at a fast rate by using low-energy plasma-enhanced chemical vapour deposition (CVD) ${ }^{142}$. As a result of this forward-grading process, the mobility in modulation-doped $\mathrm{Ge} / \mathrm{SiGe}$ was enhanced to above $10^{5} \mathrm{~cm}^{2}(\mathrm{Vs})^{-1}\left(\mathrm{REFS}^{143,144}\right)$.

Later on, the advent of reverse-graded relaxed SiGe buffers ${ }^{145,146}$ with improved threading dislocation density $\left(\sim 10^{6} \mathrm{~cm}^{-2}\right)$ was a turning point in the development of $\mathrm{Ge} / \mathrm{SiGe}$ heterostructures. High-quality relaxed $\mathrm{Ge}$ was grown directly on a Si substrate by reduced-pressure $\mathrm{CVD}^{147-149}$, followed by reverse grading of the Ge composition in the SiGe buffers. Ultra-high hole mobility exceeding $10^{6} \mathrm{~cm}^{2}(\mathrm{Vs})^{-1}$ was achieved in a modulation-doped strained $\mathrm{Ge}$ quantum well at $T<10 \mathrm{~K}\left(\mathrm{REF}^{8}{ }^{8}\right.$ ). A key enabler for the order-of-magnitude improvement in mobility was the near-perfect epitaxy attainable with an industrial reduced-pressure CVD tool, with a very low background of ionized impurities (such as boron, aluminium and gallium) and defects in the Ge quantum well and the surrounding SiGe epilayers.

This exceptional mobility put a spotlight on $\mathrm{Ge} / \mathrm{SiGe}$ as a platform for quantum devices. Previous studies on $\mathrm{Si}-\mathrm{SiGe}$ heterostructures showed that undoped structures are better suited for the operation of QDs and spin qubits because ionized impurities in the doping layer may cause leakage paths, parasitic channels and charge noise ${ }^{150-152}$. This critical know-how was directly ported to the Ge/SiGe platform and efforts were concentrated on undoped, accumulation-only heterostructures. As a result, the development of quantum devices in planar Ge was greatly accelerated. A high mobility of $5 \times 10^{5} \mathrm{~cm}^{2}(\mathrm{Vs})^{-1}$ at $T=1.7 \mathrm{~K}$ was measured in $\mathrm{Ge} / \mathrm{SiGe}$ heterostructure field-effect transistors $(\mathrm{H}-\mathrm{FETs})^{9}$ using an industry-compatible, high- $\kappa$ dielectric, setting a new benchmark for holes in shallow, buried-channel transistors. Further improvements are expected by an engineered optimization of the stack and device fabrication parameters, such as strain in the quantum well, barrier thickness ${ }^{46,153,154}$ and dielectric-deposition conditions.

The superior quality achieved in reverse-graded $\mathrm{Ge} / \mathrm{SiGe}$ heterostructures enabled a plethora of quantum-transport studies in modulation-doped, etched Hall-bar devices ${ }^{90,155-169}$ and, more recently, in undoped H-FETs ${ }^{46,91,153,154,170-173}$. Initial expectations from band-structure considerations were confirmed and the understanding of confined holes in planar Ge was advanced. Very light in-plane effective masses were measured both in modulation-doped Hall bars $\left(0.055 m_{0}\right)^{167}$ and in undoped H-FETs $\left(0.061 m_{0}\right){ }^{46}$ for devices aligned with the $<110\rangle$ crystallographic direction. The mass is further reduced to $0.035 m_{0}$ for devices aligned along the $<100>$ direction $^{167}$. Magnetotransport studies ${ }^{9,168,172}$ confirmed that $2 \mathrm{D}$ holes in strained Ge exhibit large, effective $g$-factors for an out-of-plane $\boldsymbol{B}$, with a reported value $^{172}$ of $g_{\perp} \approx 20$ at a density below $1 \times 10^{11} \mathrm{~cm}^{-2}$. Large spin-splitting energies (up to $\approx 1 \mathrm{meV}$ ) were observed $^{89-91,159,161,162,165,174}$ due to a cubic Rashba-type spin-orbit interaction. As a single-band system with large Zeeman energy, the 2DHG in high-mobility strained $\mathrm{Ge}$ is an interesting system in which to investigate fractional quantum Hall physics ${ }^{163,166,169}$ (FIG. 2b), with fractional states observed even above $4 \mathrm{~K}$. Furthermore, the ability to control the carrier density within a single $\mathrm{H}$-FET has given insights into the percolation threshold density to establish a metallic $2 \mathrm{D}$ channel in Ge. A very low value of $\sim 2 \times 10^{10} \mathrm{~cm}^{-2}$ was reported ${ }^{154}$, indicative of very low disorder in the low-density regime relevant for spin qubits in QDs.

One-dimensional hole channels and quantum point contacts were the first proof-of-principle devices fabricated by electrostatic confinement of 2DHGs in undoped strained $\mathrm{Ge} / \mathrm{SiGe}$ heterostructures ${ }^{175-177}$. The high quality of the heterostructures, with hole mean free paths on the order of a micron, enabled the observation of quantum ballistic transport in $1 \mathrm{D}$ channels as long as $600 \mathrm{~nm}$ (REF. ${ }^{176}$ ) (FIG. 2C), clean quantized conductance plateaus ${ }^{175,176}$ and, possibly, charge fractionalization due to strong interactions at low densities ${ }^{177}$. Furthermore, a large $g$-factor anisotropy was observed $\left(g_{\perp}>10\right.$ and $\left.g_{\|}<1\right)$, confirming the $\mathrm{HH}$ character of the dominant valence-band states imparted by the strong vertical confinement.

\section{Nanowires}

Ge/Si core-shell nanowires. The Ge/Si core-shell NW heterostructure has a type II band alignment with a valence-band offset of about $500 \mathrm{meV}$, and holes are strongly confined in the Ge core ${ }^{178}$. The development of $\mathrm{Ge} / \mathrm{Si}$ core-shell NWs traces back to the growth of Si and Ge NWs by the vapour-liquid-solid (VLS) mechanism using laser-ablated clusters of Au with nanometre diameters as catalysts ${ }^{179}$. The NWs can grow up to $30 \mu \mathrm{m}$ in length, and the metal catalysts define precisely both the position and the diameter of the NWs; diameters are in the range of 6-100 $\mathrm{nm}$ and 3-100 $\mathrm{nm}$ for Si and Ge NWs, respectively. By depositing homogeneously sized Au catalysts onto oxide-free $\mathrm{Si}$, Ge NWs vertically oriented along the [111] direction and with uniform diameter and length were obtained on $\operatorname{Si}(111)$ substrates ${ }^{180}$. Ordered arrays of Ge NWs with controlled position were demonstrated by using lithographically patterned Au nanoparticles ${ }^{181}$, and kinked Ge NW structures were reported, with iterative control over their nucleation and growth ${ }^{182}$. The NW growth rate decreases for smaller diameters, with a cut-off at sufficiently small sizes ${ }^{181}$. Furthermore, the NW growth orientation is strongly correlated with the NW diameter. Ge NWs with diameters larger than $20 \mathrm{~nm}$ are mostly oriented along the [111] direction, whereas [110] and [112] orientations are preferred for NWs with smaller diameters ${ }^{183,184}$.

The know-how accumulated in the development of $\mathrm{Si}$ and Ge NWs paved the way towards the epitaxial growth of core-shell Ge/Si NW heterostructures, which were first synthesized by the vapour-phase deposition of a conformal Si shell on a VLS-grown Ge $\operatorname{core}^{178}$. The mostly amorphous Si shell was grown at low 
temperature to form an abrupt $\mathrm{Ge} / \mathrm{Si}$ interface and was then fully crystallized by in situ thermal annealing with negligible intermixing. With elevated growth temperature and the introduction of $\mathrm{HCl}$ to avoid strain-driven surface roughing during the shell growth, high-quality Ge/Si NWs with a low density of defects and a sharp interface were realized ${ }^{183,184}$ (FIG. 3a). In addition, radial, multi-shell-doping and even modulation-doping coreshell NWs have been demonstrated ${ }^{178,185}$. Recently, an advanced radial heterostructure was synthesized with a core made of short segments of crystalline Ge and $\mathrm{Al}$ surrounded by a Si shell (Ge/Al core/Si shell NWs), opening the way to the synthesis of epitaxial S-Sm heterostructures in Ge NWs ${ }^{186}$.

Shortly after the demonstration of Ge/Si core-shell NWs, in 2005, a high-quality 1D hole gas (1DHG) was measured in Ge $\left(\right.$ REF $\left.^{187}\right)$. Controlled, single-holetransistor behaviour was observed at low temperature when the Si shell was used as a tunnel barrier to the $\mathrm{Ge}$ core. Enhanced mobility in undoped structures resulted in carrier mean free paths with signatures of ballistic transport up to a few hundred nanometres, at both low and room temperatures ${ }^{187,188}$. Further studies also revealed a dependence of the carrier mobility on the NW orientation (FIG. 3b). Compared with the conventional [111]-oriented NWs, [110]-oriented Ge/Si core-shell
NWs show substantially enhanced hole mobility, with values as high as $4,200 \mathrm{~cm}^{2}(\mathrm{Vs})^{-1}$ at $4 \mathrm{~K}\left(\mathrm{REF}^{184}\right)$.

The high quality of the Ge/Si core-shell NWs allowed for a detailed investigation of quantum transport in $1 \mathrm{DHG}$ at low temperatures. In REF. ${ }^{70}$, weak antilocalization was observed and attributed to spin-orbit coupling, which could be modulated by up to a factor five with an external electric field (FIG. 3c). More recently, detailed measurements of spin-orbit energies in $1 \mathrm{DHGs}$ in $\mathrm{Ge} / \mathrm{Si}$ $\mathrm{NWs}^{73,74}$ confirmed the large values expected from the $\mathrm{DRSOI}^{50,51}$. In REF. ${ }^{73}$, highly tunable $1 \mathrm{DHG}$ field-effect transistors with a dual-gate architecture were fabricated, and very large and tunable spin-orbit energies in the range of $1-6 \mathrm{meV}$ were measured, almost one order of magnitude larger than those reported for NWs in III-V semiconductors. A comparable spin-orbit energy of $2.1 \mathrm{meV}$ was reported in $\mathrm{REF}^{74}$, which also presented a $g$-factor of 3.6 arising from strong HH-LH mixing in the wires.

Despite the tremendous progress over the past 15 years, there are still two key materials challenges to address for further advancing the study of Ge/Si NWs hosting $1 \mathrm{DHGs}$. One is the development of catalyst-free methods to grow high-quality Ge NWs, because the use of metal catalysts inevitably introduces metal contamination in the semiconductor NWs. The other challenge a Core-shell Ge/Si NWs

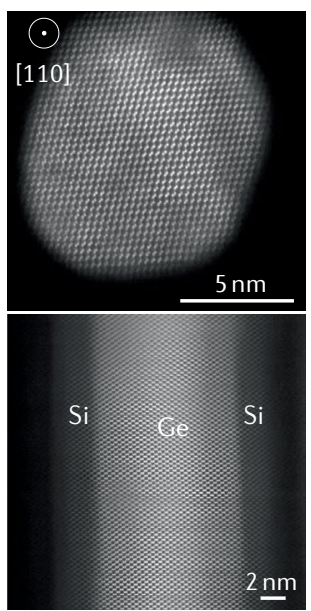

b Mobility

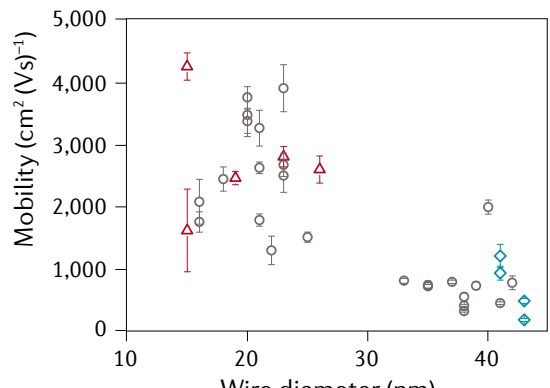

Wire diameter $(\mathrm{nm})$

Orientation not measured

$\diamond$ [111]-oriented NWs

$\Delta$ [110]-oriented NWs
C Spin-orbit coupling

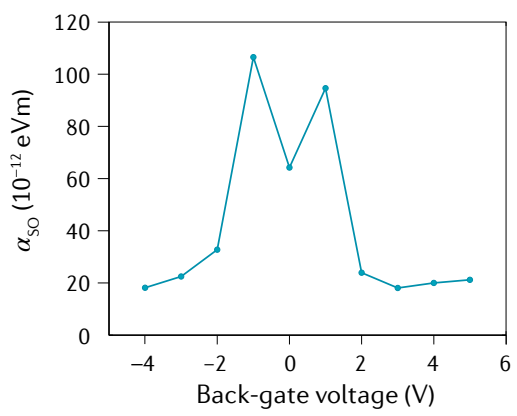

d Ge HWs

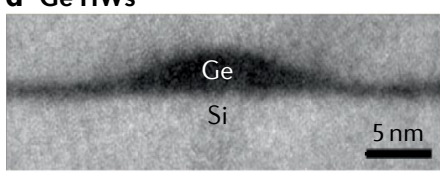

\section{e Site-controlled Ge HWs}

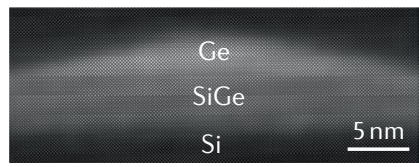

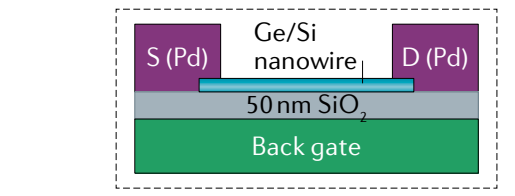

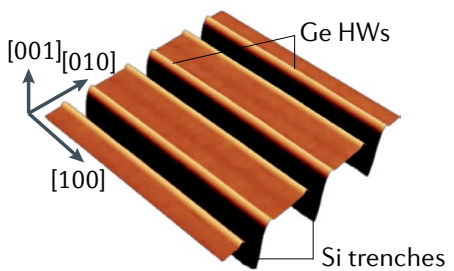

Fig. 3 | Ge-based nanowires. a | High-angle annular dark-field image of a Ge/Si core-shell nanowire (NW) along the radial (top) and axial (bottom) directions. $\mathbf{b} \mid$ Hole mobility of NWs at $4 \mathrm{~K}$ as a function of wire diameter for different NW orientations. c|Spin-orbit coupling strength, $\alpha_{\mathrm{so}}$, as a function of the back-gate voltage in a NW field-effect transistor (schematically shown in the inset). D, drain; S, source. d | Transmission electron microscopy image of a Ge hut wire (HW) on Si covered with a Si cap. e | Transmission electron microscopy (left) and atomic force microscopy (right) images of site-controlled Ge HWs with highly uniform size on trench-patterned $\mathrm{Si}$. The HWs grow on a 1D SiGe layer that forms at the [100]-oriented trench edges of the patterned Si. Panels $\mathbf{a}$ and $\mathbf{b}$ are adapted with permission from REF. ${ }^{184}$, American Chemical Society. Panel $\mathbf{c}$ is adapted with permission from REF. ${ }^{70}$, American Chemical Society. Panel $\mathbf{d}$ is adapted with permission from REF. ${ }^{193}$, American Physical Society. Panel $\mathbf{e}$ is adapted with permission from REF. ${ }^{10}$, Wiley-VCH Verlag $\mathrm{GmbH} \&$ Co. 
is how to transfer and arrange the out-of-plane NWs into large-scale in-plane configurations with fully controllable positions. Preliminary results demonstrated the controlled catalyst-free fabrication of $\mathrm{Si}-\mathrm{Ge}-\mathrm{Si}$ core-double shell nanowires hosting a $1 \mathrm{DHG}$ in the Ge layer. The NWs were grown using a combination of nanoimprint lithography, etching and $\mathrm{CVD}^{189}$.

Ge hut wires. The growth of Ge HWs built upon the epitaxial growth of self-assembled $\mathrm{Ge}$ QDs on $\mathrm{Si}$, which was studied extensively in the $1990 \mathrm{~s}^{190}$. 3D self-assembled Ge QDs ${ }^{191}$ were obtained by depositing about four monolayers of strained $\mathrm{Ge}$ on a $\mathrm{Si}(001)$ substrate. The resulting Ge QDs, which had a height of about 1-2 nm and four $\{105\}$ facets with a rectangular base area, were called hut clusters. In situ scanning tunnelling microscopy studies ${ }^{192}$ showed that hut clusters grow slowly with a growth rate that decreases throughout the annealing process. In 2012, it was reported that hut clusters elongate along the in-plane [001] or [010] crystallographic directions into micrometre-long Ge HWs (FIG. 3d) under appropriate molecular-beam-epitaxy growth and annealing conditions ${ }^{193}$.

In-plane Ge HWs are uniform, with an average height of about $2 \mathrm{~nm}$. They have the hut clusters' characteristic four $\{105\}$ facets and a shallow triangular cross section with a side inclination angle of $11.3^{\circ}$. This cross section is completely different from that of VLS-grown cylindrical NWs, and more resembles that of a laterally confined quasi-2D system. The planar density of HWs can be controlled simply by the amount of the initially deposited Ge. A slightly increased amount of Ge leads to a high density of short HWs. By contrast, less Ge results in a reduced nucleation rate $^{194}$ and, thus, in a low density of HWs up to $2 \mu \mathrm{m}$ long, with a large length-to-height aspect ratio of up to $\approx 1,000$.

Ge HWs have a constant height and width along the wire on each atomic terrace. Their height increases or decreases by one atomic layer when the wire crosses an atomic step. Thus, by choosing the morphology of the underlying Si surface, homogeneous or tapered Ge HWs can be obtained ${ }^{193,195}$. The further deposition of an epitaxial layer of $\mathrm{Si}$ a few nanometres thick at low temperature $\left(350^{\circ} \mathrm{C}\right)$ is sufficient to fully strain the Ge HWs and produce a sharp Si/Ge interface. Transistor-type devices made from single wires can support large current densities $\left(10^{7} \mathrm{~A} \mathrm{~cm}^{-2}\right)$ at low temperature ${ }^{193}$. The hole channel can be pinched off and, near pinch-off, single-hole transport can be observed due to the Coulomb blockade at temperatures below a few K $\left(\right.$ REF. $\left.^{193}\right)$. So far, mobility and quantum-transport studies in single Ge HWs are very limited compared with those on $2 \mathrm{DHG}$ in $\mathrm{Ge} / \mathrm{SiGe}$ and on $1 \mathrm{DHG}$ in $\mathrm{Ge} / \mathrm{Si} \mathrm{NWs}$.

Although uniform in-plane Ge HWs can be grown without a catalyst, the random position of the HWs on the Si substrate is a bottleneck for the scale-up of quantum devices. Recent work has addressed this challenge by exploiting the self-assembled growth of $\mathrm{Ge}$ on a lithographically patterned $\mathrm{Si}$ substrate, which resulted in the site-controlled growth of highly uniform in-plane $\mathrm{Ge}$ HWs on $\mathrm{Si}(001)$ substrates $^{10}$ (FIG. 3e). The Ge HWs grow selectively on an initially formed 1D SiGe layer at the [100]-oriented trench edges of patterned Si. They have a height of about $3.8 \mathrm{~nm}$, with a standard deviation of merely $0.11 \mathrm{~nm}$, and their position, distance and length can be precisely controlled. As shown in the transmission electron microscopy image in FIG. 3e, sharp Ge/SiGe and $\mathrm{Si} / \mathrm{Ge}$ interfaces form. In addition, closely spaced parallel HWs and even square-shaped or L-shaped structures consisting of four or two Ge HWs can be obtained.

\section{Germanium quantum devices}

Core-shell NWs, HWs and planar heterostructures have emerged in the past 15 years as the materials platforms of choice to demonstrate the basic building blocks for Ge-based quantum information processing. Results obtained with QDs, spin qubits and S-Sm hybrids hold promise for the development of a fast and scalable $\mathrm{Ge}$ quantum technology that can potentially rely on hybrid combinations of semiconductors, superconductors and topological phases of matter.

\section{Quantum dots}

Low-dimensional systems such as planar heterostructures and NWs are naturally suited for QD experiments (FIG. 4). In these systems with geometrical confinement to one or two dimensions, additional confinement is required to create QDs, that is, $0 \mathrm{D}$ islands. Gate electrodes isolated via suitable dielectrics and/or Schottky barriers can be used for the tunnel barriers defining the QD and its connections to the source, drain and gate electrodes. The experimental demonstration of gate-controlled Ge QDs has allowed the investigation of spin states, $g$-factors and Pauli spin blockade through low-temperature magnetotransport and charge-sensing measurements.

Nanowires. The first report of a $1 \mathrm{DHG}$ in Ge/Si core-shell NWs included the observation of Coulomb-blockade oscillations due to single-hole tunnelling from the contacts through the thin Si shell into the Ge core ${ }^{187}$. Regular Coulomb diamonds were observed, indicating low disorder in the wire. Such wires were then combined with local gate electrodes to create double QDs and integrated charge sensors ${ }^{11}$. These experiments demonstrated the possibility to control the tunnel coupling, albeit in the multi-hole regime. Further advances in materials quality ${ }^{184}$ enabled more tunable QDs ${ }^{196}$, allowing to define single, double and triple QDs in Ge/Si NWs (FIG. 4a) with a low hole-occupation number ${ }^{197}$ (FIG. 4d). Transport measurements in NW QDs have been used to probe the spin states of holes, and, for strongly confined QDs, an effective $g$-factor of $\sim 2$ has been measured ${ }^{198}$. Nonetheless, a strong anisotropy was predicted ${ }^{101}$ and measured $^{72}$ to be $g_{\max } / g_{\min } \approx 13$ in NW QDs. This large anisotropy follows from an almost vanishing effective $g$-factor $\left(g_{\min }=0.2\right)$ along the NW axis. Finally, Pauli spin blockade can elucidate the spin-flip mechanisms through the behaviour of the leakage current as a function of magnetic field ${ }^{197,199,200}$.

Hut wires. As for $\mathrm{Ge} / \mathrm{Si} \mathrm{NWs}$, the first report on $\mathrm{Ge} \mathrm{HWs}$ included the observation of single-hole transport ${ }^{193}$. However, the Coulomb diamonds observed in the HWs were more irregular than those in Ge/Si NWs, 


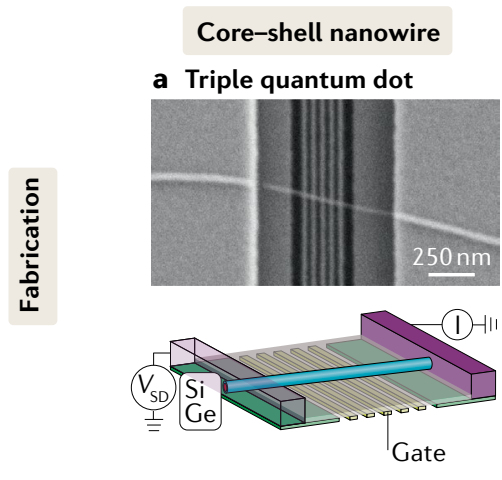

d Triple-dot resonances

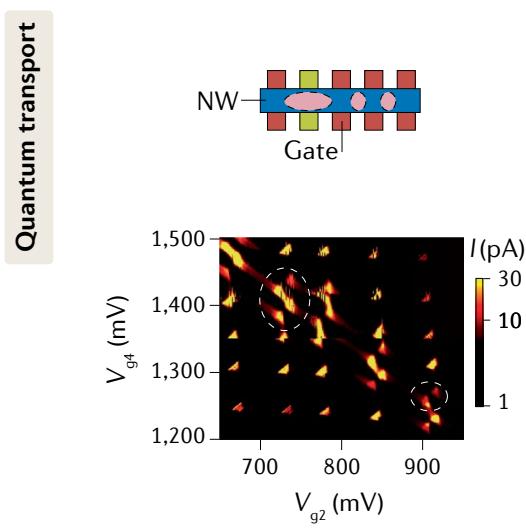

g Spin dephasing

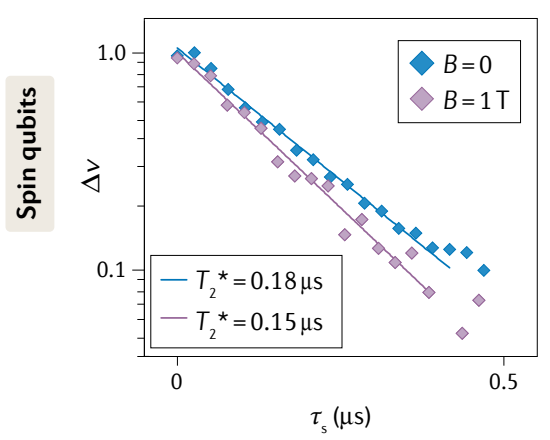

Hut wire

b Double quantum dot

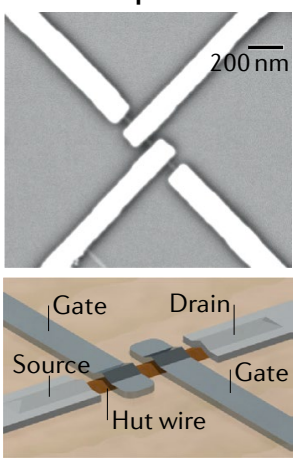

e Charge sensing

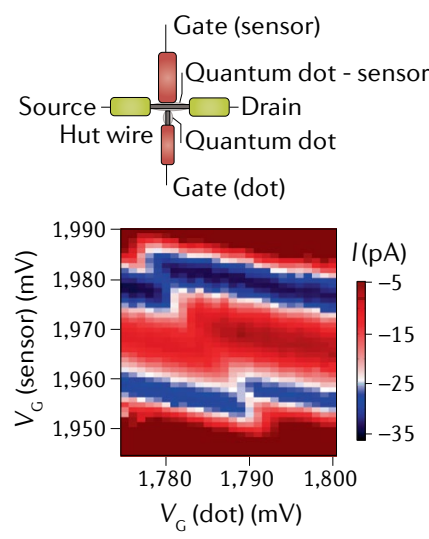

h Qubit control

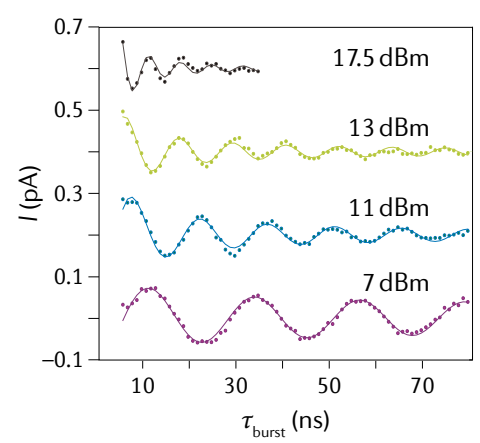

Planar heterostructure

c Quantum-dot array
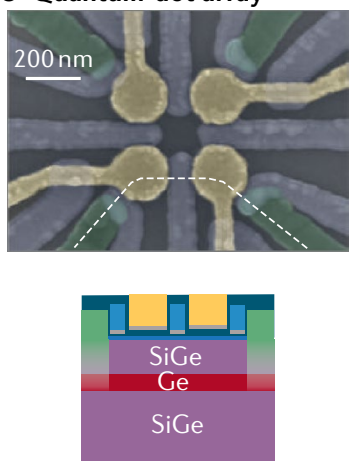

f Single-hole occupancy
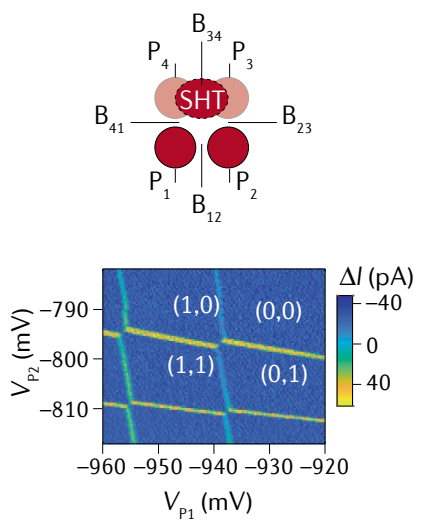

i Fast two-qubit logic

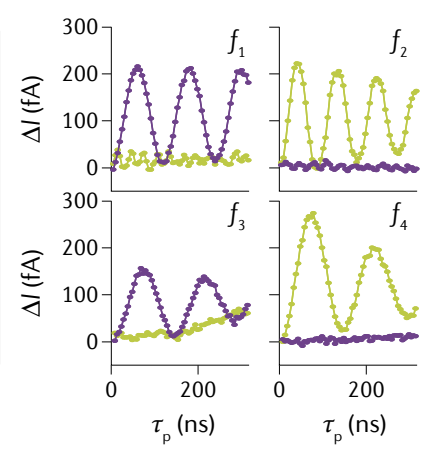

owing to disorder ${ }^{193}$. Subsequent improvements in materials and fabrication processes led to the observation also in this system of gate-controlled single and double QDs ${ }^{14,15,201}$ (FIG. 4b) and of radio frequency (rf) charge sensing ${ }^{202}$ (FIG. 4e). Spin states and $g$-factors can be measured in QDs via ground-state and excited-state magnetospectroscopy ${ }^{203}$. The hole band structure often leads to anisotropic $g$-factors. In HWs, the lowest-energy states have mostly $\mathrm{HH}$ nature, and large anisotropies of $16-18$ with $\mathrm{HH} g$-factors of up to 4.4 were found in HW QDs ${ }^{14}$.

Planar heterostructures. After high-quality undoped $\mathrm{Ge} / \mathrm{SiGe}$ heterostructures ${ }^{9}$ became available, remarkable advances were achieved with planar QDs, and, in only two years, this platform matured from demonstrations of gate-controlled single QDs ${ }^{18}$ to quadruple QDs arranged in a $2 \mathrm{D}$ array ${ }^{204}$ (FIG. 4C), highlighting the benefits of planar systems for scalability. Owing to the high mobility ${ }^{9}$ and light mass of holes ${ }^{46}$, comparatively large QDs (with a diameter of $\approx 100 \mathrm{~nm}$ ) can be defined ${ }^{18}$ and tuned to contain only a single hole ${ }^{21,204}$ in Ge/SiGe quantum wells. Furthermore, these devices are compatible with electric-gate fabrication using single-layer technology ${ }^{18}$. Moreover, these QDs can be directly contacted by aluminium leads, resulting in a completely dopant-free architecture $^{18}$. The low disorder of the host heterostructures results in QDs that are electrically stable and show excellent homogeneity ${ }^{18}$ and limited charge noise, and QDs with charge noise values $\sqrt{S_{\mathrm{E}}}<0.2 \mu \mathrm{eV} / \sqrt{\mathrm{Hz}}$ at $1 \mathrm{~Hz}$, which are below the measurement-detection limit, have been reported ${ }^{154}$. Double QDs have also been 
4 Fig. 4 Quantum dots and qubits in core-shell nanowires, hut wires and planar systems. a | Triple quantum dots have been realized in nanowires (NWs). $\mathbf{b} \mid$ Double quantum dots in hut wires. c | Quadruple quantum dots in planar systems. The dotted line indicates the cross section shown in the schematic, with plunger gates (yellow) defining the quantum dots tunnel-coupled to metallic leads (green). $\mathbf{d}$ | Triple-dot stability diagram of a NW measured in transport. A honeycomb pattern of current resonances - the signature of a double quantum dot - is observed, distorted by interference with a third dot (dashed circles). e | Charge sensing performed with a single quantum dot in a hut wire. f |Charge sensing single-hole occupancy in a reconfigurable quadruple quantum dot in planar $\mathrm{Ge}$. The four quantum dots are defined using plunger gates $\mathrm{P}$ and coupled through barrier gates $\mathrm{B}$. A single-hole transistor (SHT) is defined by strongly coupling two quantum dots and functions as a sensor for the other quantum-dot pair, as shown in the stability diagram, where reaching the last hole is clearly demonstrated. $\mathbf{g}$ | Fast pulsing in NWs can be used to measure spin relaxation and coherence. The normalized differential voltage, $\Delta v$, versus separation time, $\tau_{\mathrm{s}}$, is plotted for two different values of the magnetic field. The dashed and solid lines are exponential fits, yielding the dephasing times $T_{2}^{*}$ in the legend. The similar timescales at $B=0$ and $B=1 T$ may be the result of either spin-orbit coupling or interaction with the bath of nuclear spins in the atomic lattice. $\mathbf{h}$ | Electric dipole spin resonance enables fast driving in Ge hut wires. Current, I, versus microwave burst time, $\tau_{\text {burst }}$, curves for radio frequency power in the range $7-17.5 \mathrm{dBm}$ show Rabi oscillations. A Rabi frequency approaching $150 \mathrm{MHz}$ is observed at the highest power (black curve). i | By combining single-qubit and two-qubit gates, universal quantum logic was demonstrated in planar Ge. Electric dipole spin resonance driving enables the execution of controlled rotation operation and the two-qubit CX gate can be executed in a timescale of $55 \mathrm{~ns}$. The purple and green curves show the conditional rotation of the target qubit for the two possible states of the control qubit. The four quadrants correspond to the four resonance frequencies present in an exchange-coupled qubit pair. Panels $\mathbf{a}$ and $\mathbf{d}$ are adapted with permission from REF. ${ }^{197}$. American Institute of Physics. Panels $\mathbf{b}$ and $\mathbf{h}$ are adapted with permission from REF. ${ }^{15}$, Springer Nature Limited, with inclusion of additional data (panel $\mathbf{h}$, black curve $17.5 \mathrm{dBm}$ ). Panels $\mathbf{c}$ and $\mathbf{f}$ are adapted with permission from REF. ${ }^{204}$, American Institute of Physics. Panel $\mathbf{e}$ is adapted with permission from REF. ${ }^{202}$, American Chemical Society. Panel $\mathbf{g}$ is adapted with permission from REF. ${ }^{206}$, American Chemical Society. Panel $\mathbf{i}$ is adapted with permission from REF. ${ }^{19}$, Springer Nature Limited. reported $^{19,173,205} \cdot g_{\|}$of $0.2-0.3$ were measured consistently in QDs in planar $\mathrm{Ge}^{18,19,205}$, together with an anisotropy $g_{\perp} / g_{\|} \approx 18$. This large anisotropy denotes the $\mathrm{HH}$ character of the first sub-bands, as expected in the limit of strong confinement along the growth axis. In planar Ge QDs, a remarkable control over the inter-dot tunnel coupling and detuning has been obtained ${ }^{19}$, enabling operation at the charge-symmetry point for optimized quantum control ${ }^{19}$. Furthermore, single-hole occupation has been achieved ${ }^{21,204}, \mathrm{rf}$ charge sensing was realized in quadruple QDs ${ }^{204}$ (FIG. 4f) and $2 \times 2$ QD grids - stepping stones towards $2 \mathrm{D}$ arrays - were operated.

\section{Spin qubits}

Spin relaxation and dephasing (this and other useful terms are defined in BOX 1) has been measured in $\mathrm{Ge} / \mathrm{Si}$ core-shell $\mathrm{NWs}^{206}$, single-qubit rotations have been performed in Ge $\mathrm{HWs}^{15}$ and single-qubit operation with a single-hole spin $^{21}$ and two-qubit logic has been executed in planar structures ${ }^{19}$ (FIG. 5). As measured experimentally ${ }^{15,18-21}$, Ge hole-spin qubits exhibit large excited states, greatly exceeding the valley-splitting energies in $\mathrm{Si}$, resulting in well-defined qubit states. The key challenge for quantum information is to increase the number of qubits and to scale in two dimensions. In other systems like electrons in $\mathrm{Si}$, coherent control of single spins requires large objects such as microwave striplines or nanomagnets, and it is an open question as to how to integrate them with a larger number of exchange-coupled qubits ${ }^{207}$. The spin-orbit coupling of holes in Ge offers an opportunity to circumvent this bottleneck and facilitate scalability. Nonetheless, it also opens new sources of decoherence. In this section, we review quantum coherence and provide perspectives for future experiments.

The spin lifetime and coherence can be measured by exploiting the exchange interaction in a double QD to prepare suitable spin states (for example, spin singlets or triplets), or by Rabi driving the spin to the desired states using microwave pulses, and monitoring their time evolution. Spin lifetimes have been measured in Ge nanowires using fast gate pulsing and tuning the exchange interaction (FIG. $4 \mathrm{~g}$ ). A spin lifetime $T_{1}=0.6 \mathrm{~ms}$ has been measured in the zero-magnetic-field limit, which reduces to $T_{1}=0.2 \mathrm{~ms}$ at $1 \mathrm{~T}\left(\right.$ REF. $\left.^{12}\right)$. These results have been obtained using transport measurements for an unknown number of confined holes estimated to be between 10 and 50. By integrating charge sensors and barrier gates to control the tunnel rates, isolated single spins in $\mathrm{Ge}$ can be measured; a spin lifetime $T_{1}=32 \mathrm{~ms}\left(\mathrm{REFS}^{21,208}\right)$ in a magnetic field $B=0.67 \mathrm{~T}$ was obtained for planar QDs. Ramsey sequences have been used to measure the spin dephasing times, yielding $T_{2}^{*}=0.18 \mu$ s for $\mathrm{Ge} / \mathrm{Si}$ core-shell $\mathrm{NWs}^{206}, T_{2}^{*}=0.13 \mu \mathrm{s}$ for Ge HWs ${ }^{15}$ and $T_{2}^{*}=0.82 \mu \mathrm{s}$ for planar Ge (REFS $\left.{ }^{19,21}\right)$. Interestingly, the measured dephasing time in planar Ge significantly exceeds $T_{2}^{*}=270 \mathrm{~ns}$ reported for holes in $\mathrm{Si}\left(\mathrm{REF}^{209}\right)$. Although the origin of dephasing is under investigation and may be different for each platform, it has been observed that the dephasing time is related to the resonance-frequency dependence on the electric field ${ }^{21}$. This suggests that charge noise, which can couple to the spin-qubit states via spin-orbit coupling, is an important factor. Reducing the charge noise or reducing the spin-orbit coupling, therefore, are possible strategies to optimize the quantum coherence. In addition, the quantum coherence can be extended via dynamical decoupling; this way, a coherence time $T_{2}=100 \mu$ s has been reported ${ }^{20}$.

Holes in Ge can be all-electrically controlled by electric dipole spin resonance ${ }^{29,30}$, as has been demonstrated in $\mathrm{Ge} \mathrm{NWs}^{210}, \mathrm{HWs}^{15}$ and Ge planar structures ${ }^{19,21}$. Fast Rabi driving speeds exceeding $100 \mathrm{MHz}$ have been obtained by simply applying electric tones to the same gates that are used to define the QDs ${ }^{15,19}$. The first $\mathrm{Ge}$ qubit was realized in a double QD in Ge HWs, with readout performed using Pauli spin blockade in transport ${ }^{15}$. By fixing the gate voltages to the Pauli spin-blockade regime and by sweeping the microwave frequency while stepping the magnetic field, an increase in the current was observed when the condition $h f=g \mu_{\mathrm{B}} B$ was fulfilled. This current increase is due to the fact that the microwave field induces to a spin rotation via electric dipole spin resonance. From the slope of the electric dipole spin resonance line, the $g$-factor can be extracted and its anisotropy as a function of the magnetic field can be studied. In the case of different $g$-factor values in the two QDs, two lines can be observed ${ }^{19}$. To demonstrate coherent qubit rotations, the QD system is pulsed to Coulomb blockade, and then a short rf burst is applied and pulsed back to the Pauli spin-blockade regime for readout. By changing the duration of the rf burst, coherent spin 
oscillations can be realized (FIG. 4h). A particular challenge in transport measurements is that the current amplitude depends on the timescale of the experiment, $\delta I=2 e / t_{\text {cycle }}$. For typical cycle times $t_{\text {cycle }} \approx 1 \mu \mathrm{s}$, the resulting current is only a fraction of a pA. To obtain high-quality data, lock-in techniques have been used together with reference pulses to compensate for slow variations in the offset current ${ }^{19}$.

By coherently controlling both spins in a planar $\mathrm{Ge}$ double QD, the two-qubit Hilbert space can be assessed by combining single-qubit rotations and a two-qubit controlled rotation (CROT) gate ${ }^{19}$ (FIG. 4i). The individual qubit fidelities have been measured using randomized benchmarking ${ }^{211}$. In this protocol, the fidelity decay is monitored as a function of a series of randomly drawn Clifford gates. An average single-qubit fidelity $F_{\mathrm{C}}=99.3 \%$ has been observed. The two-qubit logic is then obtained by tuning the exchange interaction. This can be done via the detuning energy in the presence of finite tunnel coupling or by directly controlling the tunnel coupling. The latter method has proven advantageous, as it allows to operate at the charge-symmetry point, where the system is, to a first-order approximation, insensitive to detuning noise ${ }^{122,123,212}$. The advantage of this strategy is clear if we look at the strong dependence of quantum coherence on detuning ${ }^{19}$, which suggests significant detuning noise, most likely due to charge noise. In this context, the advantage of low disorder and low effective mass in planar Ge becomes evident, as it allows to incorporate a dedicated barrier gate to tune to the charge-symmetry point while having substantial exchange interaction. In this regime, algorithms have been performed to demonstrate the coherence of the two-qubit logic with $\mathrm{CZ}$ gates executed on timescales of only $12 \mathrm{~ns}\left(\mathrm{REF}^{20}{ }^{20}\right.$. By performing single-qubit randomized benchmarking, fidelities of up to $99.9 \%$ have been achieved ${ }^{20}$. Extracting the two-qubit fidelity requires techniques such as two-qubit randomized benchmarking and has yet to be demonstrated.

\section{Superconductor-semiconductor hybrids}

In S-Sm hybrids ${ }^{213,214}$, the semiconducting component of the device acquires superconducting properties from the superconductor, a phenomenon known

\section{Box 1 | Glossary}

Ramsey sequence: Measurement to probe the dephasing time. The sequence consists of a $\pi / 2$ pulse to rotate a spin state from the ground state into superposition, a waiting time to monitor the phase coherence and a final $\pi / 2$ pulse to rotate to the basis state, allowing for measurement.

Clifford gates: All gates that bring a qubit state from one point in the Clifford group to another point in the Clifford group, corresponding to permutations of the Pauli operations.

CROT: The two-qubit logic gate, where the target qubit is rotated conditionally dependent on the state of the control qubit.

$C X$ gate: Two-qubit logic gate, where the target qubit makes a $\pi$ rotation conditionally dependent on the state of the control qubit.

CZ gate: Two-qubit logic gate, where the target qubit makes a precession of $\pi$ conditionally dependent on the state of the control qubit.

$T_{1}$ : Relaxation time (the time it takes to relax from the excited state to the ground state). $T_{2}^{*}$ : Dephasing time (the time before phase is incoherent during free precession). as the superconducting proximity effect. As a result, a device with a short semiconducting section connecting two superconducting electrodes can behave as a superconducting Josephson junction with a critical current, $I_{c}$, tuned by a gate voltage. Such a device, known as a $\mathrm{JoFET}^{215}$, can be integrated in a superconducting 'transmon' qubit. In the resulting device, named gatemons, the qubit characteristic energy is electrostatically tuned via the JoFET gate voltage ${ }^{5,216,217}$. Gatemons are of practical interest for large-scale integration. Their tuning relies on gate voltages and not on $\mathrm{mA}$-scale currents flowing through inductively coupled circuits, which can help minimize crosstalks and limit power dissipation along the control lines. Hybrid S-Sm structures are also highly relevant for topological quantum computation based on MZMs. These exotic quasiparticle states have been predicted to emerge at the edges of a 1D S-Sm system, provided the semiconductor has strong spin-orbit coupling and a sufficiently large magnetic field is applied along certain directions ${ }^{214}$.

Most experimental research has been focusing on hybrid S-Sm systems involving small-band-gap III-V semiconductors such as InAs and InSb. Germanium is an appealing alternative semiconductor to host hybrid S-Sm devices and enable their large-scale integration on Si. In Ge-based S-Sm hybrids, the favoured semiconductor quasiparticle states are holes and not electrons. In fact, multiple experiments have shown that the Fermi energy of certain superconducting metals can pin close to the valence-band edge of $\mathrm{Ge}$, resulting in a highly transparent $\mathrm{S}-\mathrm{Sm}$ interface.

The first experimental demonstration of a JoFET in $\mathrm{Ge}$ was based on a single $\mathrm{Ge} / \mathrm{Si}$ core-shell NW connecting two superconducting $\mathrm{Al}$ electrodes ${ }^{22}$ (FIG. 6a). Well below $1 \mathrm{~K}$, that is, well below the superconducting critical temperature of the Al contacts, the device exhibited a superconducting regime with a gate-dependent critical current and an $I_{\mathrm{c}} R_{\mathrm{N}}$ product close to the ideal value, denoting a very high transparency of the contacts ( $R_{\mathrm{N}}$ is the normal-state resistance). The experimental demonstration of high-quality proximitized $\mathrm{Ge} / \mathrm{Si}$ NWs (FIG. 6b) and the recent measurements of a strong direct Rashba spin-orbit coupling ${ }^{74,75}$ make 1 DHGs in $\mathrm{Ge} / \mathrm{Si}$ core-shell NWs a favourable system for the realization of topological superconductivity and $\mathrm{MZMs}^{50,58}$. Furthermore, novel S-Sm device structures such as a linear array of superconducting QDs were proposed to realize robust and practical Majorana chains ${ }^{218}$. Ge-based superconducting QDs were pioneered in strained SiGe self-assembled nanocrystals ${ }^{95}$; the recent demonstration of superconducting QDs in Ge/Si core-shell NWs ${ }^{23,75}$ represents an important step towards these alternative architectures for MZMs.

The observation of a tunable superconducting proximity effect in $\mathrm{Ge} / \mathrm{Si}$ core-shell NWs was reproduced in more recent experiments using not only $\mathrm{Al}\left(\mathrm{REFS}^{75,186,219}\right.$ ) but also NbTiN (REF. ${ }^{188}$ ) superconducting contacts. The high transparency of the S-Sm contacts was confirmed by tunnel-spectroscopy measurements ${ }^{220}$, which revealed an induced superconducting gap containing only a small density of quasiparticle states. This experimental finding is particularly important in view of creating Majorana 


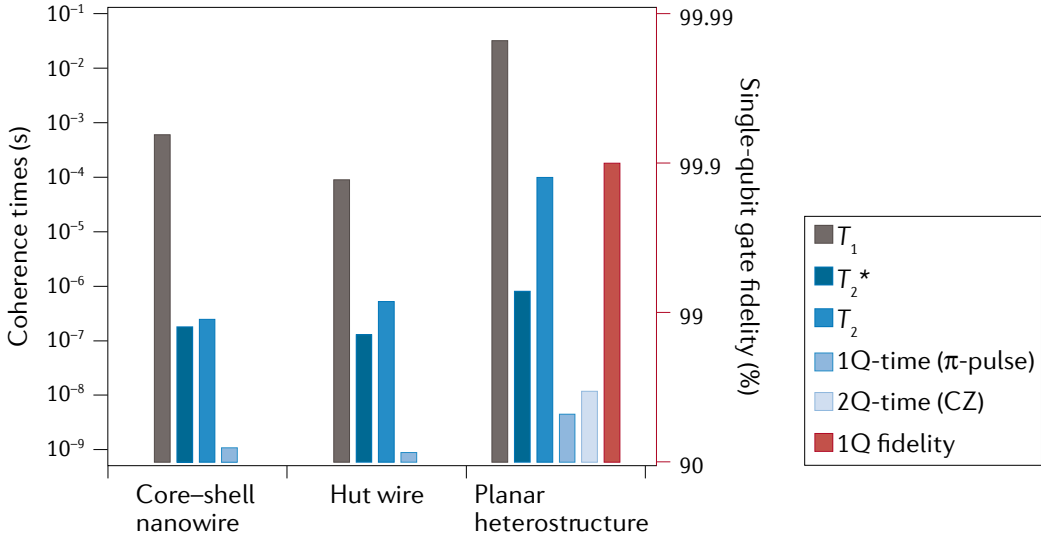

Fig. 5 | State-of-the-art coherence times and single-qubit gate fidelities for coreshell nanowires, hut wires and planar heterostructures. In each platform, spin relaxation times, $T_{1}$, dephasing times, $T_{2}^{*}$, and coherence times, $T_{2}$, have been measured, and coherent Rabi rotations with frequencies above $100 \mathrm{MHz}$ have been performed. An extended $T_{2}$ of $100 \mu$ s has been realized in Ge using dynamical decoupling, and one-qubit (1Q) fidelities nearing fault-tolerant thresholds have been measured using randomized benchmarking. Fast two-qubit logic has been executed using exchangecoupled quantum dots in planar Ge with a two-qubit (2Q) CZ gate executed on a timescale of $12 \mathrm{~ns}$. Data for nanowires are from REFS ${ }^{12,206,210}$. Data for hut wires are from REFS $^{15,201,257}$. Data for planar heterostructures are from REFS $19,20,208$.

edge states whose potentially long lifetime is not ruined by quasiparticle poisoning.

Besides Ge/Si NWs, JoFETs in Ge were recently demonstrated in accumulation-only undoped $\mathrm{Ge} / \mathrm{SiGe}$ heterostructures by contacting the induced $2 \mathrm{DHG}$ with $\mathrm{Al}$ electrodes ${ }^{18,24,221}$. Owing to the rather high hole mobility, supercurrent transport was observed even for Ge channels as long as $6 \mu \mathrm{m}\left(\mathrm{REF}^{221}\right.$ ) (FIG. 6c). Compared with $\mathrm{Ge} / \mathrm{Si}$ NWs, planar $\mathrm{Ge} / \mathrm{SiGe}$ heterostructures are a more versatile materials platform, offering ample freedom for device design. FIGURE $6 \mathrm{~d}$ shows the scanning electron microscope image of an $\mathrm{Al} / \mathrm{Ge} / \mathrm{Al}$ junction embedding an additional pair of split gates defining a quantum point contact. Upon varying the voltage on these gates, the switching current and subgap conductance exhibit a step-like structure, reflecting the discretized opening of 1D hole modes ${ }^{221}$. Another example of a hybrid S-Sm device consisting of two independently controlled JoFETs fabricated on the same mesa structure and embedded in an $\mathrm{Al}$ superconducting ring is shown in FIG. 6e $\left(\mathrm{REF}^{24}\right)$. This geometry realizes a gate-tunable, superconducting quantum-interference device.

We have already stressed the importance of having $\mathrm{S}-\mathrm{Sm}$ junctions that are highly transparent to the flow of carriers between the semiconductor and the superconductor. To this end, the possible presence of insulating interface layers, such as native oxides, needs to be avoided. The in situ deposition of the superconducting metal right after the growth of the semiconductor offers an effective solution ${ }^{222,223}$. Interestingly, Ge-based structures also allow for an equally valuable ex situ approach relying on the thermally activated propagation of the superconducting element into the $\mathrm{Ge}$ channel. This process was demonstrated for Ge-based NWs contacted by Al electrodes ${ }^{224}$. Transmission tunnelling microscopy carried out during the annealing process showed $\mathrm{Al}$ entering the $\mathrm{NW}$ and replacing
Ge (REF. $\left.{ }^{225}\right)$. This solid-state reaction results in a clean, atomically sharp $\mathrm{Al} / \mathrm{Ge}$ interface progressively moving into the Ge NW. Because of its low activation energy, this process can occur for annealing temperatures as low as $250^{\circ} \mathrm{C}$. Considering that device fabrication involves processing steps at comparably high temperatures (such as atomic-layer deposition), the inner diffusion of $\mathrm{Al}$ is probably at the origin of the high contact transparencies observed in most of the experiments we discussed. Recently, $\mathrm{Al} / \mathrm{Ge} / \mathrm{Al}$ devices with an extremely short Ge channel were obtained by a controlled annealing process ${ }^{188}$ (FIG. 6b). The application of this contact-fabrication method to other superconducting metals with higher critical temperatures and critical magnetic fields would further enlarge the range of device functionalities accessible with Ge-based hybrid systems.

\section{Outlook}

After two decades of research on quantum computation with $\mathrm{QDs}^{3}$, the ingredients for extensible qubit tiles are becoming concrete ${ }^{207,226}$ and several appealing architectures have been proposed ${ }^{227-230}$. Attractive architectures for large-scale quantum computing are based on qubit modules, consisting of linear or 2D arrays, interconnected using long-range links ${ }^{207}$. Although one may expect that the interconnections themselves will form the bottleneck, quantum error-correction schemes show that remarkable error rates can be tolerated when the qubit modules can execute quantum logic with high fidelity ${ }^{231}$. Great progress has been made with QD qubits: universal gates sets for quantum logic ${ }^{19,232}$, rudimentary quantum algorithms ${ }^{233}$, electron spin states using gate-based readout ${ }^{234-237}$, strong coupling between spin and microwave photons $\mathrm{s}^{238,239}$ and interactions between distant electron spins ${ }^{240}$ have been demonstrated. The grand challenge is to integrate these individual elements on a single platform to build scalable quantum technology. In this respect, Ge is particularly appealing, because it may allow for the cointegration of different quantum components relying on spin, superconductivity or, possibly, topologically protected quasiparticle states, as illustrated in FIG. 7. Here, we present an outline of these possible quantum components and identify some of the exciting pursuits that lay ahead for $\mathrm{Ge}$ quantum electronics based on NWs, HWs and planar systems.

\section{Electrically driven logical qubits}

The all-electric control of hole spins mediated by spinorbit coupling has already led to the operation of a universal gate set, obtained by combining single-quibit ${ }^{15,19}$ and two-qubit logic ${ }^{19}$. It, furthermore, provides a pathway to scale hole QD systems in two dimensions, as required for quantum error correction ${ }^{241}$. As opposed to electron spin qubits, which require large objects such as striplines or magnets to coherently control the electron $\operatorname{spin}^{207}$, hole QDs have all elements for operation intrinsically available. This can largely facilitate scalability in two dimensions. However, as for all spin qubits relying on either intrinsic or synthetic spin-orbit coupling, Ge-based qubits are susceptible to a fairly significant variability due to the inherent dependence of hole $g$-factors 
a NW Josephson transistor
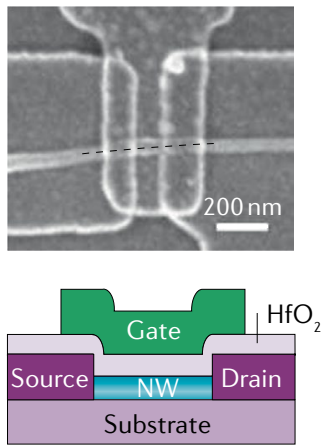

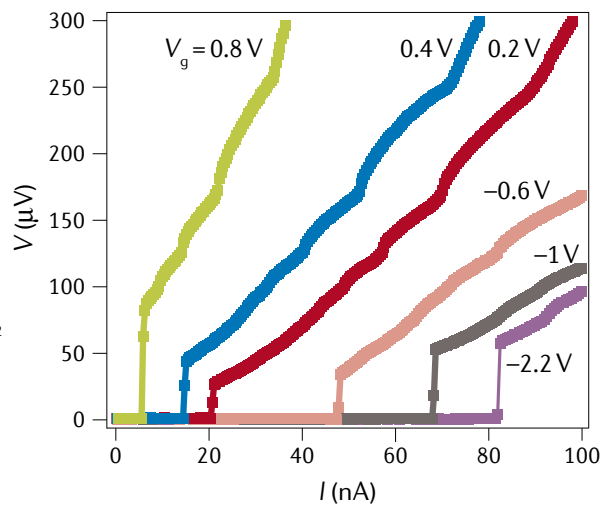

d Super-quantum point contact b Abrupt S-Sm interface

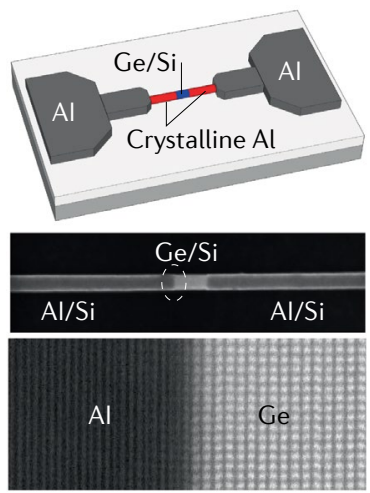

e Interferometer
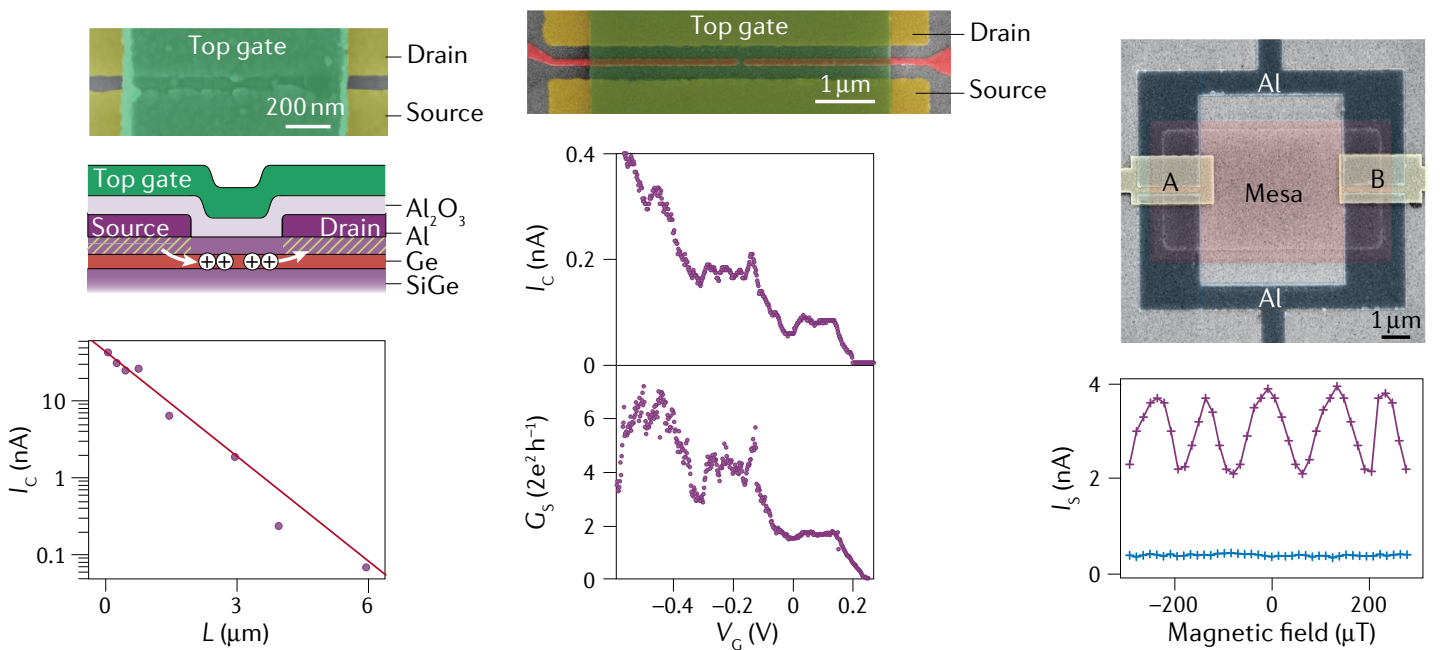

Fig. 6 | Superconductor-semiconductor hybrids in Ge nanowires and planar systems. a | Josephson junctions have been realized in both Ge/Si core-shell nanowires (NWs), shown here, and in planar Ge/SiGe, shown in panel c. In Josephson junctions, the switching current - denoting the transition from the superconducting state to the normal state - depends on the gate voltage, $V_{g}$, as can be seen on the voltage-current $(V-l)$ traces measured at $60 \mathrm{mK}$. b $\mid$ Atomically abrupt superconductor-semiconductor (S-Sm) interfaces have been engineered in an axial Al/Ge/Al NW heterostructure with an ultra-thin Si shell wrapped around it. c-e | In planar systems, supercurrent transport has been observed over $\mu \mathrm{m}$-long channels, and superconducting quantum point contacts and interferometers have been fabricated. $\mathbf{c} \mid$ Length $(L)$ dependence of the supercurrent, $I_{c}$, in planar systems (such as the ones shown in the schematic and scanning electron microscope image). A purely exponential decay is observed with a decay length of $1.0 \mu \mathrm{m}$, and supercurrents have been measured over unprecedented lengths of $6 \mu \mathrm{m}$. $\mathbf{d}$ | In superconducting quantum point contacts (such as the one shown in the scanning electron microscope image), the discretization of the switching current, $l_{\mathrm{s}}$, and of the subgap conductance, $G_{s}$, as a function of gate voltage, $V_{G}$, have been reported, demonstrating ballistic transport. e / Superconducting quantum interference devices, such as the one shown in the image, consist of two Josephson junctions (A and B) positioned in the two arms of a superconducting ring. Periodic oscillations in the switching current $I_{\mathrm{S}}$ (purple curve) are observed as a function of the out-of-plane magnetic field when both junctions are turned on. $I_{\mathrm{S}}$ does not oscillate (blue curve) if one junction is off. Panel $\mathbf{a}$ is reproduced with permission from REF. ${ }^{22}$, Springer Nature Limited. Panel $\mathbf{b}$ is reproduced with permission from REF. ${ }^{186}$, American Chemical Society. Panel c (top) is adapted from REF. ${ }^{18}$, Springer Nature Limited. Panels c (bottom) and $\mathbf{d}$ are adapted with permission from $\mathrm{REF}^{221}$, American Physical Society. Panel $\mathbf{e}$ is reproduced with permission from REF. ${ }^{24}$, American Chemical Society.

on the QD confinement potential. This variability issue will need to be accounted for in the development of scalable architectures. Nevertheless, first demonstrations of 2D QD arrays in Ge have already been reported ${ }^{204}$. Furthermore, Ge can be purified to contain only isotopes with zero nuclear spins to remove nuclear spin bath dephasing and allow for long quantum coherence times ${ }^{107,242}$. This holds great promise to engineer QD systems in which quantum error-correction schemes can be implemented to build logical qubits with an error rate below that of the physical qubits.

\section{Gate-based readout of single-hole spins}

The strong spin-orbit coupling of holes also provides a natural advantage for gate-based readout of single spins. Research from several groups has shown the promise of this approach using electron spins in Si (REFS $\left.{ }^{234-236}\right)$. Adapting this technology to holes in Ge may further 

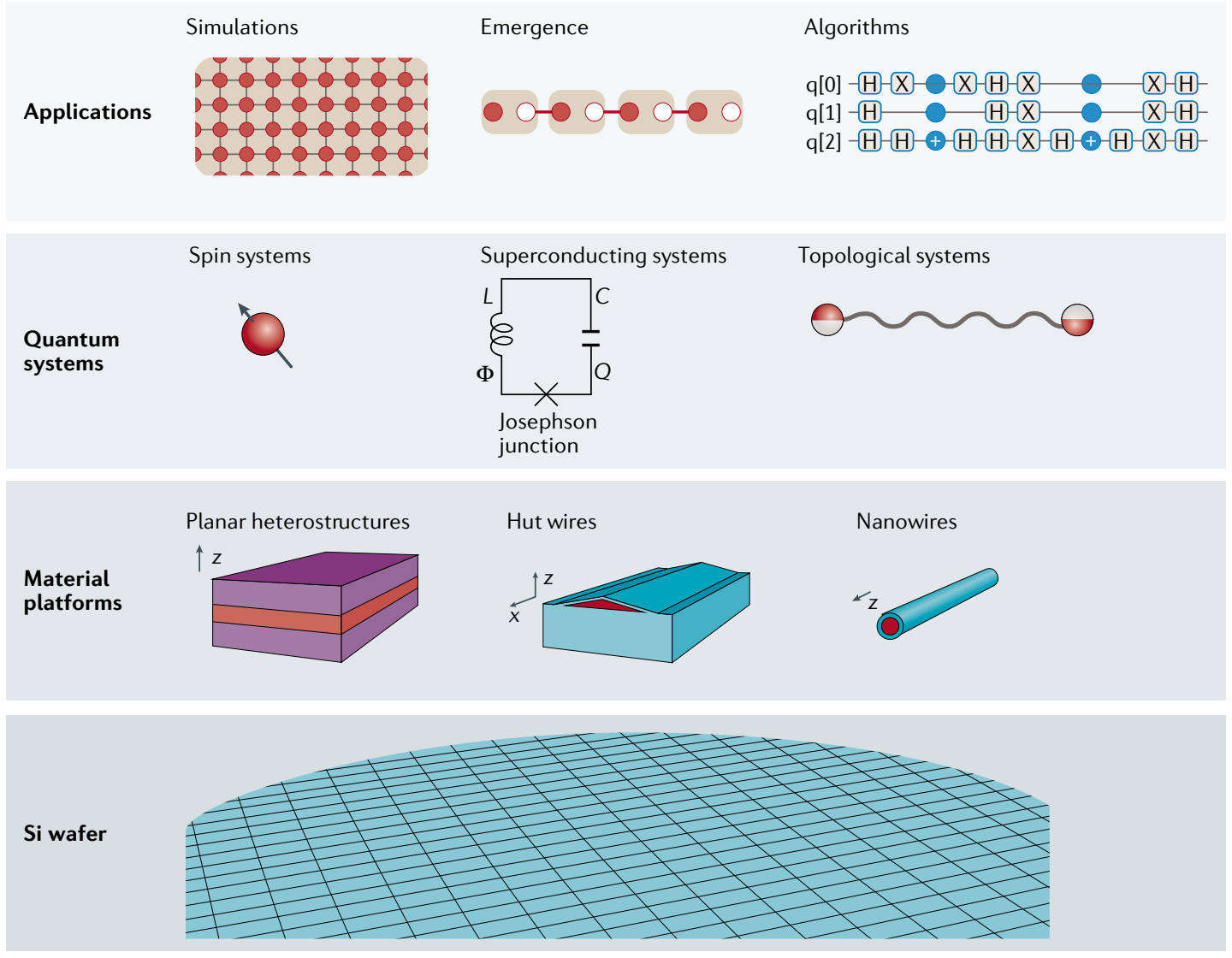

Fig. 7 | Ge-based quantum technology. Ge is an extremely rich platform that supports the development of spin, superconducting and topological systems. Each of these systems defines an exciting direction that may support high-fidelity qubits and quantum logic, and their cointegration in Ge provides an opportunity for scalable quantum technology on a Si wafer. Hybrid quantum systems offer a unique platform for the simulation of non-trivial Hamiltonians, such as the FermiHubbard Hamiltonian, and allow for the controllable emulation of emergent phenomena, such as Majorana zero modes in $p$-wave superconductors. For fault-tolerant quantum computation, topological regions may function as error-protected long-range links for spin qubits, whereas single-qubit rotations on gatemons or spin qubits can complement operations on topological qubits to define a universal quantum gate set.

increase the readout rate, as well as its fidelity. First steps in this direction have already been taken by showing fast rf readout ${ }^{204,205}$ and capacitive coupling ${ }^{16}$.

\section{Spin-spin long-distance entanglement}

Single-hole spins may be coherently coupled to microwave photons using on-chip superconducting resonators. Strong spin-orbit coupling can act as an efficient mediator to achieve spin-photon coupling ${ }^{243}$, with rates beyond those achieved with electrons ${ }^{238,239}$, and first steps towards such coupling seem encouraging ${ }^{16}$. By coupling two hole spins to a single resonator and bringing them into resonance, one can entangle hole qubits that are far apart. Such a result would be a milestone towards the realization of extensible qubit tiles coupled via long-range links.

\section{Superconducting gatemons}

Another advantage of holes in Ge is that many metals have a Fermi-level pinning to the valence band of Ge (REF. ${ }^{6}$ ), meaning that Schottky-barrier-free contacts can be made to superconductors. Gate-tunable superconductivity has been reported for $\mathrm{NWs}^{22}$ and planar systems $^{18}$, and superconducting quantum-interference devices have already been realized ${ }^{24}$. This opens exciting perspectives to build gatemons ${ }^{5,216}$. Gatemons may form the basis of a scalable qubit tile, in particular, when based on $2 \mathrm{D}$ systems $\mathrm{s}^{217}$, but they could also function as a readout mechanism and as long-range links to couple distant hole-spin qubits.

\section{Topological qubits based on MZMs}

Unconventional superconductivity has been studied for a long time ${ }^{244}$ but gained renewed attention in the past ten years after the prediction that exotic superconductivity may emerge in S-Sm systems ${ }^{245,246}$. In particular, the combination of strong spin-orbit coupling, magnetic field and superconductivity may lead to superconductivity with $p$-wave symmetry, providing an experimental test bed to emulate the original Kitaev chain ${ }^{4}$. In contrast to the common Bardeen-CooperSchrieffer $s$-wave superconductors, superconductors with $p$-wave symmetry may host zero-energy states that can be described as Majorana fermions. These states could exhibit non-Abelian exchange statistics ${ }^{247}$ and form the basis of topological quantum computation ${ }^{248}$. 
Although several exciting experiments have been performed using InAs and InSb nanostructures ${ }^{214}$, definite proof of the existence of isolated MZMs has yet to be provided. Holes in Ge can exhibit strong spin-orbit coupling, and Ge quantum devices may, therefore, form an excellent host for MZMs ${ }^{56,58}$. Furthermore, by introducing a novel Berry phase ${ }^{249}$, heavy holes may add a new twist to Majorana states.

\section{Quantum information transfer between different qubit types}

Germanium turns out to be an exciting material to explore several types of qubits. It also provides the opportunity to study the interaction between different qubits. Perhaps the most exciting one is the interaction between topologically trivial and topological non-trivial qubits. Quantum information transfer may occur between spin qubits based on individual holes and parity qubits based on MZMs ${ }^{250-253}$. Apart from being very interesting scientifically, such a transfer may also resolve key challenges in quantum information. First, it could provide a topological link between separated hole qubits. Second, it may be used to construct a universal gate set for topological qubits. Topological qubits based on MZMs can only be used to construct states within the Clifford group, which can be efficiently simulated on a classical computer ${ }^{248}$. The quantum gate set may be enriched by exploiting the spin-qubit toolbox to enable full universal logic. Such a realization would represent a formidable achievement in topological quantum computing, but significant challenges remain. Nonetheless, exciting intermediate steps can be expected along the way. These include the coupling of superconductors to spin qubits to enable superconductivity-mediated long-range coupling between spin qubits based on crossed Andreev reflection ${ }^{254,255}$; the interaction is predicted to work on length scales exceeding micrometres in $1 \mathrm{D}$ systems ${ }^{256}$.

Published online: 21 December 2020
1. Pillarisetty, R. Academic and industry research progress in germanium nanodevices. Nature $\mathbf{4 7 9}$, 324-328 (2011)

2. Kamata, Y. High-k/Ge MOSFETs for future nanoelectronics. Mater. Today 11, 30-38 (2008).

3. Loss, D. \& DiVincenzo, D. P. Quantum computation with quantum dots. Phys. Rev. A 57, 120-126 (1998).

4. Kitaev, A. Y. Unpaired Majorana fermions in quantum wires. Phys.-Uspekhi 44, 131-136 (2001).

5. Larsen, T. et al. Semiconductor-nanowire-based superconducting qubit. Phys. Rev. Lett. 115, 127001 (2015).

6. Dimoulas, A., Tsipas, P., Sotiropoulos, A. \& Evangelou, E. K. Fermi-level pinning and charge neutrality level in germanium. Appl. Phys. Lett. 89, 252110 (2006)

7. Pillarisetty, R. et al. in Proceedings of the 2018 IEEE International Electron Devices Meeting (IEDM) 6.3.1-6.3.4 (IEEE, 2018).

8. Dobbie, A. et al. Ultra-high hole mobility exceeding one million in a strained germanium quantum well. Appl. Phys. Lett. 101, 172108 (2012).

9. Sammak, A. et al. Shallow and undoped germanium quantum wells: a playground for spin and hybrid quantum technology. Adv. Funct. Mater. 29, 1807613 (2019).

10. Gao, F. et al. Site-controlled uniform Ge/Si hut wires with electrically tunable spin-orbit coupling. Adv. Mater. 32, 1906523 (2020)

11. Hu, Y. et al. A Ge/Si heterostructure nanowire-based double quantum dot with integrated charge sensor. Nat. Nanotechnol. 2, 622-625 (2007).

12. Hu, Y., Kuemmeth, F., Lieber, C. M. \& Marcus, C. M Hole spin relaxation in $\mathrm{Ge}-\mathrm{Si}$ core-shell nanowire qubits. Nat. Nanotechnol. 7, 47-50 (2012).

13. Ares, N. et al. Nature of tunable hole $g$ factors in quantum dots. Phys. Rev. Lett. 110, 046602 (2013).

14. Watzinger, $\mathrm{H}$. et al. Heavy-hole states in germanium hut wires. Nano Lett. 16, 6879-6885 (2016).

15. Watzinger, $\mathrm{H}$. et al. A germanium hole spin qubit. Nat. Commun. 9, 3902 (2018)

16. Xu, G. et al. Dipole coupling of a hole double quantum dot in germanium hut wire to a microwave resonator. New J. Phys. 22, 083068 (2020).

17. Li, Y. et al. Coupling a germanium hut wire hole quantum dot to a superconducting microwave resonator. Nano Lett. 18, 2091-2097 (2018).

18. Hendrickx, N. W. et al. Gate-controlled quantum dots and superconductivity in planar germanium. Nat. Commun. 9, 2835 (2018).

19. Hendrickx, N., Franke, D., Sammak, A., Scappucci, G. $\&$ Veldhorst, M. Fast two-qubit logic with holes in germanium. Nature 577, 487-491 (2020).

20. Hendrickx, N. W. et al. A four-qubit germanium quantum processor. Preprint at arXiv http://arxiv.org/ abs/2009.04268 (2020).

21. Hendrickx, N. W. et al. A single-hole spin qubit Nat. Commun. 11, 3478 (2020).
22. Xiang, J., Vidan, A., Tinkham, M., Westervelt, R. M $\&$ Lieber, C. M. Ge/Si nanowire mesoscopic Josephson junctions. Nat. Nanotechnol. 1, 208-213 (2006).

23. Ridderbos, J. et al. Josephson effect in a few-hole quantum dot. Adv. Mater. 30, 1802257 (2018).

24. Vigneau, F. et al. Germanium quantum-well Josephson field-effect transistors and interferometers. Nano Lett. 19, 1023-1027 (2019)

25. Petta, J. R. et al. Coherent manipulation of coupled electron spins in semiconductor quantum dots. Science 309, 2180-2184 (2005).

26. Koppens, F. H. L. et al. Driven coherent oscillations of a single electron spin in a quantum dot. Nature 442, 766-771 (2006).

27. Nowack, K. C. Koppens, F. H. L., Nazarov, Y. V. \& Vandersypen, L. M. K. Coherent control of a single electron spin with electric fields. Science $\mathbf{3 1 8}$, 1430-1433 (2007).

28. Kloeffel, C. \& Loss, D. Prospects for spin-based quantum computing in quantum dots. Annu. Rev. Condens. Matter Phys. 4, 51-81 (2013).

29. Bulaev, D. V. \& Loss, D. Spin relaxation and decoherence of holes in quantum dots. Phys. Rev. Lett 95, 076805 (2005).

30. Bulaev, D. V. \& Loss, D. Electric dipole spin resonance for heavy holes in quantum dots. Phys. Rev. Lett. 98, 097202 (2007).

31. Fischer, J., Coish, W. A., Bulaev, D. V. \& Loss, D. Spin decoherence of a heavy hole coupled to nuclear spins in a quantum dot. Phys. Rev. B 78, 155329 (2008).

32. Trif, M., Simon, P. \& Loss, D. Relaxation of hole spins in quantum dots via two-phonon processes. Phys. Rev. Lett. 103, 106601 (2009).

33. Heiss, D. et al. Observation of extremely slow hole spin relaxation in self-assembled quantum dots. Phys. Rev. B 76, 241306 (2007).

34. Gerardot, B. D. et al. Optical pumping of a single hole spin in a quantum dot. Nature 451, 441-444 (2008).

35. Brunner, D. et al. A coherent single-hole spin in a semiconductor. Science 325, 70-72 (2009).

36. Warburton, R. J. Single spins in self-assembled quantum dots. Nat. Mater. 12, 483-493 (2013)

37. Nolting, W. \& Ramakanth, A. Quantum Theory of Magnetism (Springer, 2009)

38. Winkler, R. Spin-Orbit Coupling Effects in Two-Dimensional Electron and Hole Systems (Springer, 2003)

39. Luttinger, J. M. \& Kohn, W. Motion of electrons and holes in perturbed periodic fields. Phys. Rev. 97, 869-883 (1955)

40. Luttinger, J. M. Quantum theory of cyclotron resonance in semiconductors: general theory. Phys. Rev. 102, 1030-1041 (1956).

41. Winkler, R., Culcer, D., Papadakis, S. J., Habib, B. \& Shayegan, M. Spin orientation of holes in quantum wells. Semicond. Sci. Technol. 23, 114017 (2008).

42. Lawaetz, P. Valence-band parameters in cubic semiconductors. Phys. Rev. B 4, 3460-3467 (1971).
43. Terrazos, L. A. et al. Theory of hole-spin qubits in strained germanium quantum dots. Preprint at arXiv http://arxiv.org/abs/1803.10320 (2020).

44. Bir, G. L. \& Pikus, G. E. Symmetry and Strain-Induced Effects in Semiconductors (Wiley, 1974).

45. van Kesteren, H. W., Cosman, E. C., van der Poel, W. A. J. A. \& Foxon, C. T. Fine structure of excitons in type-II GaAs/AIAs quantum wells. Phys. Rev. B 41 , 5283-5292 (1990).

46. Lodari, M. et al. Light effective hole mass in undoped $\mathrm{Ge} / \mathrm{SiGe}$ quantum wells. Phys. Rev. B 100, 041304 (2019).

47. Sercel, P. C. \& Vahala, K. J. Analytical formalism for determining quantum-wire and quantum-dot band structure in the multiband envelope-function approximation. Phys. Rev. B 42, 3690-3710 (1990).

48. Harada, Y., Kita, T., Wada, O. \& Ando, H. Anisotropic magneto-optical effects in one-dimensional diluted magnetic semiconductors. Phys. Rev. B 74, 245323 (2006).

49. Csontos, D., Brusheim, P., Zülicke, U. \& Xu, H. Q. Spin- $-\frac{3}{2}$ physics of semiconductor hole nanowires: Valence-band mixing and tunable interplay between bulk-material and orbital bound-state spin splittings. Phys. Rev. B 79, 155323 (2009).

50. Kloeffel, C., Trif, M. \& Loss, D. Strong spin-orbit interaction and helical hole states in $\mathrm{Ge} / \mathrm{Si}$ nanowires. Phys. Rev. B 84, 195314 (2011).

51. Kloeffel, C., Rančić, M. J. \& Loss, D. Direct Rashba spin-orbit interaction in $\mathrm{Si}$ and Ge nanowires with different growth directions. Phys. Rev. B 97, 235422 (2018).

52. Menéndez, J., Singh, R. \& Drucker, J. Theory of strain effects on the Raman spectrum of Si-Ge core-shell nanowires. Ann. Phys. 523, 145-156 (2011).

53. Kloeffel, C., Trif, M. \& Loss, D. Acoustic phonons and strain in core/shell nanowires. Phys. Rev. B 90 115419 (2014).

54. Kloeffel, C. Trif, M. Stano, P \& Loss, D. Circuit OED with hole-spin qubits in $\mathrm{Ge} / \mathrm{Si}$ nanowire quantum dots. Phys. Rev. B 88, 241405 (2013).

55. Nigg, S. E., Fuhrer, A. \& Loss, D. Superconducting grid-bus surface code architecture for hole-spin qubits. Phys. Rev. Lett. 118, 147701 (2017).

56. Maier, F., Meng, T. \& Loss, D. Strongly interacting holes in Ge/Si nanowires. Phys. Rev. B 90, 155437 (2014).

57. Mao, L., Gong, M., Dumitrescu, E., Tewari, S. \& Zhang, C. Hole-doped semiconductor nanowire on top of an s-wave superconductor: a new and experimentally accessible system for Majorana fermions. Phys. Rev. Lett. 108, 177001 (2012)

58. Maier, F., Klinovaja, J. \& Loss, D. Majorana fermions in Ge/Si hole nanowires. Phys. Rev. B 90, 195421 (2014).

59. Ivchenko, E. L, Kaminski, A. Y \& Rössler, U. Heavy-light hole mixing at zinc-blende $(001)$ interfaces under normal incidence. Phys. Rev. B 54, 5852-5859 (1996). 
60. Vervoort, L., Ferreira, R. \& Voisin, P. Effects of interface asymmetry on hole subband degeneracies and spin-relaxation rates in quantum wells. Phys. Rev. B 56, R12744-R12747 (1997).

61. Guettler, T. et al. Optical polarization relaxation in $\ln _{x} \mathrm{Ga}_{1-x}$ As-based quantum wells: Evidence of the interface symmetry-reduction effect. Phys. Rev. B 58 R10179-R10182 (1998).

62. Vervoort, L., Ferreira, R. \& Voisin, P. Spin-splitting of the subbands of InGaAs-InP and other 'no common atom' quantum wells. Semicond. Sci. Technol. 14, 227-230 (1999).

63. Olesberg, J. T. et al. Interface contributions to spin relaxation in a short-period InAs/GaSb superlattice. Phys. Rev. B 64, 201301 (2001).

64. Hall, K. C. et al. Spin relaxation in (110) and (001) InAs/GaSb superlattices. Phys. Rev. B 68, 115311 (2003).

65. Golub, L. E. \& Ivchenko, E. L. Spin splitting in symmetrical SiGe quantum wells. Phys. Rev. B 69 115333 (2004)

66. Nestoklon, M. O., Ivchenko, E. L., Jancu, J.-M. \& Voisin, P. Electric field effect on electron spin splitting in SiGe/Si quantum wells. Phys. Rev. B 77, 155328 (2008).

67. Prada, M., Klimeck, G. \& Joynt, R. Spin-orbit splittings in Si/SiGe quantum wells: from ideal $\mathrm{Si}$ membranes to realistic heterostructures. New J. Phys. 13, 013009 (2011)

68. Furthmeier, S. et al. Enhanced spin-orbit coupling in core/shell nanowires. Nat. Commun. 7, 12413 (2016).

69. Wojcik, P., Bertoni, A. \& Goldoni, G. Enhanced Rashba spin-orbit coupling in core-shell nanowires by the interfacial effect. Appl. Phys. Lett. 114, 073102 (2019).

70. Hao, X.-J. et al. Strong and tunable spin-orbit coupling of one-dimensional holes in $\mathrm{Ge} / \mathrm{Si}$ core/shell nanowires. Nano Lett. 10, 2956-2960 (2010).

71. Higginbotham, A. P. et al. Antilocalization of coulomb blockade in a Ge/Si nanowire. Phys. Rev. Lett. 112, 216806 (2014)

72. Brauns, M., Ridderbos, J., Li, A., Bakkers, E. P. A. M \& Zwanenburg, F. A. Electric-field dependent $g$-factor anisotropy in Ge-Si core-shell nanowire quantum dots. Phys. Rev. B 93, 121408 (2016)

73. Wang, R., Deacon, R. S., Yao, J., Lieber, C. M. \& Ishibashi, K. Electrical modulation of weakantilocalization and spin-orbit interaction in dual gated $\mathrm{Ge} / \mathrm{Si}$ core/shell nanowires. Semicond. Sci. Technol. 32, 094002 (2017)

74. Sun, J. et al. Helical hole state in multiple conduction modes in $\mathrm{Ge} / \mathrm{Si}$ core/shell nanowire. Nano Lett. 18 6144-6149 (2018)

75. de Vries, F. K. et al. Spin-orbit interaction and induced superconductivity in a one-dimensiona hole gas. Nano Lett. 18, 6483-6488 (2018).

76. Golovach, V. N., Borhani, M. \& Loss, D. Electricdipole-induced spin resonance in quantum dots. Phys. Rev. B 74, 165319 (2006)

77. Stano, P. et al. $g$-factor of electrons in gate-defined quantum dots in a strong in-plane magnetic field. Phys. Rev. B 98, 195314 (2018)

78. Camenzind, L. C. et al. Hyperfine-phonon spin relaxation in a single-electron GaAs quantum dot. Nat. Commun. 9, 3454 (2018).

79. Stano, P. et al. Orbital effects of a strong in-plane magnetic field on a gate-defined quantum dot. Phys. Rev. B 99, 085308 (2019).

80. Winkler, R. Rashba spin splitting in two-dimensional electron and hole systems. Phys. Rev. B 62 4245-4248 (2000)

81. Chesi, S., Giuliani, G. F., Rokhinson, L. P., Pfeiffer, L. N. $\&$ West, K. W. Anomalous spin-resolved point-contact transmission of holes due to cubic Rashba spin-orbit coupling. Phys. Rev. Lett. 106, 236601 (2011).

82. Nichele, F. et al. Characterization of spin-orbit interactions of $\mathrm{GaAs}$ heavy holes using a quantum point contact. Phys. Rev. Lett. 113, 046801 (2014).

83. Nichele, F. et al. Spin-orbit splitting and effective masses in $p$-type GaAs two-dimensional hole gases. Phys. Rev. B 89, 081306 (2014).

84. Miserev, D. S. \& Sushkov, O. P. Dimensional reduction of the Luttinger Hamiltonian and $g$-factors of holes in symmetric two-dimensional semiconductor heterostructures. Phys. Rev. B 95, 085431 (2017).

85. Srinivasan, A. et al. Detection and control of spin-orbit interactions in a GaAs hole quantum point contact. Phys. Rev. Lett. 118, 146801 (2017).

86. Hung, J.-T., Marcellina, E., Wang, B., Hamilton, A. R. $\&$ Culcer, D. Spin blockade in hole quantum dots: Tuning exchange electrically and probing Zeeman interactions. Phys. Rev. B 95, 195316 (2017).
87. Marcellina, E., Hamilton, A. R., Winkler, R. \& Culcer, D. Spin-orbit interactions in inversion-asymmetric two-dimensional hole systems: a variational analysis Phys. Rev. B 95, 075305 (2017)

88. Liu, H., Marcellina, E., Hamilton, A. R. \& Culcer, D. Strong spin-orbit contribution to the hall coefficient of two-dimensional hole systems. Phys. Rev. Lett. 121, 087701 (2018).

89. Mizokuchi, R. et al. Hole weak anti-localization in a strained-Ge surface quantum well. Appl. Phys. Lett. 111, 063102 (2017)

90. Moriya, R. et al. Cubic Rashba spin-orbit interaction of a two-dimensional hole gas in a strained-Ge/SiGe quantum well. Phys. Rev. Lett. 113, 086601 (2014)

91. Chou, C.-T. et al. Weak anti-localization of two-dimensional holes in germanium beyond the diffusive regime. Nanoscale 10, 20559-20564 (2018).

92. Nenashev, A. V., Dvurechenskii, A. V. \& Zinovieva, A. F. Wave functions and $g$ factor of holes in Ge/Si quantum dots. Phys. Rev. B 67, 205301 (2003).

93. Pryor, C. E. \& Flatté, M. E. Landé $g$ factors and orbital momentum quenching in semiconductor quantum dots. Phys. Rev. Lett. 96, 026804 (2006).

94. van Bree, J. et al. Anisotropy of electron and hole $g$ tensors of quantum dots: an intuitive picture based on spin-correlated orbital currents. Phys. Rev. B 93 035311 (2016).

95. Katsaros, G. et al. Hybrid superconductorsemiconductor devices made from self-assembled SiGe nanocrystals on silicon. Nat. Nanotechnol. 5 458-464 (2010)

96. Wimbauer, T., Oettinger, K., Efros, A. L., Meyer, B. K. $\&$ Brugger, H. Zeeman splitting of the excitonic recombination in $\operatorname{In}_{x} \mathrm{Ga}_{1-x}$ As/GaAs single quantum wells. Phys. Rev. B 50, 8889-8892 (1994).

97. Durnev, M. V. et al. Magnetic field induced valence band mixing in [111] grown semiconductor quantum dots. Phys. Rev. B 87, 085315 (2013).

98. Drichko, I. L. et al. In-plane magnetic field effect on hole cyclotron mass and $g_{z}$ factor in high-mobility $\mathrm{SiGe/Ge/SiGe} \mathrm{structures.} \mathrm{Phys.} \mathrm{Rev.} \mathrm{B} \mathrm{90,} 125436$ (2014).

99. Simion, G. E. \& Lyanda-Geller, Y. B. Magnetic field spectral crossings of Luttinger holes in quantum wells. Phys. Rev. B 90, 195410 (2014)

100. Pingenot, J., Pryor, C. E. \& Flattê, M. E. Electric-field manipulation of the Lande $g$ tensor of a hole in an $\mathrm{In}_{0.5} \mathrm{Ga}_{0.5} \mathrm{As} / \mathrm{GaAs}$ self-assembled quantum dot. Phys. Rev. B 84, 195403 (2011).

101. Maier, F., Kloeffel, C. \& Loss, D. Tunable $g$ factor and phonon-mediated hole spin relaxation in $\mathrm{Ge} / \mathrm{Si}$ nanowire quantum dots. Phys. Rev. B 87, 161305 (2013).

102. Ares, N. et al. SiGe quantum dots for fast hole spin Rabi oscillations. Appl. Phys. Lett. 103, 263113 (2013).

103. Marcellina, E. et al. Electrical control of the Zeeman spin splitting in two-dimensional hole systems. Phys. Rev. Lett. 121, 077701 (2018).

104. Crippa, A. et al. Electrical spin driving by $g$-matrix modulation in spin-orbit qubits. Phys. Rev. Lett. 120 , 137702 (2018).

105. Venitucci, B., Bourdet, L., Pouzada, D. \& Niquet, Y.-M Electrical manipulation of semiconductor spin qubits within the $g$-matrix formalism. Phys. Rev. B 98 155319 (2018).

106. DiVincenzo, D. P. The physical implementation of quantum computation. Fortschr. Phys. 48, 771-783 (2000).

107. Itoh, K. et al. High purity isotopically enriched $70 \mathrm{Ge}$ and $74 \mathrm{Ge}$ single crystals: isotope separation, growth, and properties. J. Mater. Res. 8, 1341-1347 (1993).

108. Asen-Palmer, M. et al. Thermal conductivity of germanium crystals with different isotopic compositions. Phys. Rev. B 56, 9431-9447 (1997).

109. Becker, P., Pohl, H.-J., Riemann, H. \& Abrosimov, N. Enrichment of silicon for a better kilogram. Phys. Status Solidi A 207, 49-66 (2010).

110. Tyryshkin, A. M. et al. Electron spin coherence exceeding seconds in high-purity silicon. Nat. Mater 11, 143-147 (2012)

111. Veldhorst, M. et al. An addressable quantum dot qubit with fault-tolerant control-fidelity. Nat. Nanotechnol. 9, 981-985 (2014)

112. Muhonen, J. T. et al. Storing quantum information for 30 seconds in a nanoelectronic device. Nat Nanotechnol 9 986-991 (2014).

113. Sigillito, A. J. et al. Electron spin coherence of shallow donors in natural and isotopically enriched germanium. Phys. Rev. Lett. 115, 247601 (2015).
114. Fischer, J. \& Loss, D. Hybridization and spin decoherence in heavy-hole quantum dots. Phys. Rev. Lett. 105, 266603 (2010).

115. Maier, F. $\&$ Loss, D. Effect of strain on hyperfineinduced hole-spin decoherence in quantum dots. Phys. Rev. B 85, 195323 (2012).

116. Zwanenburg, F. A. et al. Silicon quantum electronics. Rev. Mod. Phys. 85, 961-1019 (2013).

117. Vandersypen, L. M. K. \& Eriksson, M. A. Quantum computing with semiconductor spins. Phys. Today 72 38-45 (2019).

118. Burkard, G., Loss, D. \& DiVincenzo, D. P. Coupled quantum dots as quantum gates. Phys. Rev. B 59, 2070-2078 (1999).

119. Weiss, K. M., Elzerman, J. M., Delley, Y. L., Miguel-Sanchez, J. \& Imamoglu, A. Coherent two-electron spin qubits in an optically active pair of coupled InGaAs quantum dots. Phys. Rev. Lett. 109, 107401 (2012).

120. Chesi, S. et al. Single-spin manipulation in a double quantum dot in the field of a micromagnet. Phys. Rev. B 90, 235311 (2014)

121. Wong, C. H., Eriksson, M. A., Coppersmith, S. N. \& Friesen, M. High-fidelity singlet-triplet $S-T_{-}$qubits in inhomogeneous magnetic fields. Phys. Rev. B 92 045403 (2015)

122. Reed, M. D. et al. Reduced sensitivity to charge noise in semiconductor spin qubits via symmetric operation Phys. Rev. Lett. 116, 110402 (2016)

123. Martins, F. et al. Noise suppression using symmetric exchange gates in spin qubits. Phys. Rev. Lett. 116, 116801 (2016).

124. Abadillo-Uriel, J. C., Eriksson, M. A., Coppersmith, S. N. \& Friesen, M. Enhancing the dipolar coupling of a $S-T_{0}$ qubit with a transverse sweet spot. Nat. Commun. 10, 5641 (2019).

125. Khaetskii, A. V. \& Nazarov, Y. V. Spin-flip transitions between Zeeman sublevels in semiconductor quantum dots. Phys. Rev. B 64, 125316 (2001).

126. Golovach, V. N., Khaetskii, A. \& Loss, D. Phonon-induced decay of the electron spin in quantum dots. Phys. Rev. Lett. 93, 016601 (2004).

127. Stano, P. \& Fabian, J. Theory of phonon-induced spin relaxation in laterally coupled quantum dots. Phys. Rev. Lett. 96, 186602 (2006).

128. Kornich, V., Kloeffel, C. \& Loss, D. Phonon-assisted relaxation and decoherence of singlet-triplet qubits in Si/SiGe quantum dots. Quantum 2, 70 (2018).

129. Li, J., Venitucci, B. \& Niquet, Y.-M. Hole-phonon interactions in quantum dots: Effects of phonon confinement and encapsulation materials on spin-orbit qubits. Phys. Rev. B 102, 075415 (2020).

130. People, R. \& Bean, J. C. Band alignments of coherently strained GeSi/Si heterostructures on 00 GeSi substrates. Appl. Phys. Lett. 48, 538-540 (1986).

131. People, R. Indirect band gap and band alignment for coherently strained bulk alloys on germanium (001) substrates. Phys. Rev. B 34, 2508-2510 (1986).

132. Virgilio, M. \& Grosso, G. Type-I alignment and direct fundamental gap in SiGe based heterostructures. J. Phys Condens. Matter 18, 1021-1031 (2006).

133. Giorgioni, A. et al. Strong confinement-induced engineering of the $\mathrm{g}$ factor and lifetime of conduction electron spins in Ge quantum wells. Nat. Commun. 7, 13886 (2016).

134. Paul, D. The progress towards terahertz quantum cascade lasers on silicon substrates. Laser Photonics Rev. 4, 610-632 (2010).

135. Matthews, J. W. \& Blakeslee, A. E. Defects in epitaxial multilayers: III. Preparation of almost perfect multilayers. J. Cryst. Growth 32, 265-273 (1976).

136. Wagner, G. R. \& Janocko, M. A. Observation of a two-dimensional hole gas in boron-doped $\mathrm{Si}_{0.5} \mathrm{Ge}_{0.5} / \mathrm{Ge}$ heterostructures. Appl. Phys. Lett. 54, 66-68 (1989).

137. Murakami, E., Etoh, H., Nakagawa, K. \& Miyao, M. High hole mobility in modulation-doped and strain controlled $\mathrm{p}-\mathrm{Si}_{0.5} \mathrm{Ge}_{0.5} / \mathrm{Ge} / \mathrm{Si}_{1-x} \mathrm{Ge}_{\chi}$ heterostructures fabricated using molecular beam epitaxy. Jpn. J. Appl. Phys. 29, L1059-L1061 (1990).

138. Murakami, E. Nakagawa, K Nishida, A \& Miyao, M. Strain-controlled Si-Ge modulation-doped FET with ultrahigh hole mobility. IEEE Electron Device Lett. 12 71-73 (1991)

139. Xie, Y. H. et al. Very high mobility two-dimensiona hole gas in $\mathrm{Si} / \mathrm{Ge}_{x} \mathrm{Si}_{1-x} / \mathrm{Ge}$ structures grown by molecular beam epitaxy. Appl. Phys. Lett. 63, 2263-2264 (1993).

140. Schäffler, F. High-mobility Si and Ge structures. Semicond. Sci. Technol. 12, 1515-1549 (1997). 
141. Lee, M. L., Fitzgerald, E. A., Bulsara, M. T., Currie, M. T. \& Lochtefeld, A Strained Si, SiCe, and Ce channels for high-mobility metal-oxide-semiconductor field-effect transistors. J. Appl. Phys. 97, 011101 (2005).

142. Isella, G. et al. Low-energy plasma-enhanced chemical vapor deposition for strained $\mathrm{Si}$ and Ge heterostructures and devices. Solid State Electron. 48, 1317-1323 (2004)

143. Känel, H. V., Kummer, M., Isella, G., Müller, E. \& Hackbarth, T. Very high hole mobilities in modulation-doped $\mathrm{Ge}$ quantum wells grown by low-energy plasma enhanced chemical vapor deposition. Appl. Phys. Lett. 80, 2922-2924 (2002).

144. Rössner, B., Chrastina, D., Isella, G. \& von Känel, H Scattering mechanisms in high-mobility strained $\mathrm{Ge}$ channels. Appl Phys. Lett 84, 3058-3060 (2004).

145. Shah, V. A. et al. Reverse graded relaxed buffers for high $\mathrm{Ge}$ content SiGe virtual substrates. Appl. Phys. Lett. 93 192103 (2008)

146. Shah, V. A., Dobbie, A., Myronov, M. \& Leadley, D. R. Reverse graded $\mathrm{SiGe} / \mathrm{Ge} / \mathrm{Si}$ buffers for high-composition virtual substrates. J. Appl. Phys. 107, 064304 (2010).

147. Colace, L. et al. Metal-semiconductor-metal nearinfrared light detector based on epitaxial Ge/Si. Appl. Phys. Lett. 72, 3175-3177 (1998).

148. Gunn, L. C. III, Capellini, G., Rattier, M. J. \& Pinguet, T. J. Methods of incorporating germanium within CMOS process. US Patent 6,887,773 (2005)

149. Hartmann, J. M. et al. Reduced pressure-chemical vapor deposition of $\mathrm{Ge}$ thick layers on $\mathrm{Si}(001)$ for 1.3-1.55- $\mu \mathrm{m}$ photodetection. J. Appl. Phys. 95, 5905-5913 (2004)

150. Lu, T. M. et al. Enhancement-mode buried strained silicon channel quantum dot with tunable lateral geometry. Appl. Phys. Lett. 99, 043101 (2011).

151. Borselli, M. G. et al. Pauli spin blockade in undoped $\mathrm{Si} / \mathrm{SiGe}$ two-electron double quantum dots. Appl. Phys. Lett. 99, 063109 (2011)

152. Maune, B. M. et al. Coherent singlet-triplet oscillations in a silicon-based double quantum dot. Nature 481, 344-347 (2012).

153. Su, Y.-H., Chuang, Y., Liu, C.-Y., Li, J.-Y. \& Lu, T.-M. Effects of surface tunneling of two-dimensional hole gases in undoped $\mathrm{Ge} / \mathrm{GeSi}$ heterostructures. Phys. Rev. Mater. 1, 044601 (2017).

154. Lodari, M. et al. Low percolation density and charge noise with holes in germanium. Mater. Quantum Technol. https://doi.org/10.1088/2633-4356/abcd82 (in the press)

155. Rößner, B., Isella, G. \& Känel, H. V. Effective mass in remotely doped Ge quantum wells. Appl. Phys. Lett. 82, 754-756 (2003)

156. Irisawa, T. et al. Hole density dependence of effective mass, mobility and transport time in strained Ge channel modulation-doped heterostructures. Appl. Phys. Lett. 82, 1425-1427 (2003).

157. Sawano, K. et al. Magnetotransport properties of Ge channels with extremely high compressive strain. Appl. Phys. Lett. 89, 162103 (2006).

158. Sawano, K. et al. Strain dependence of hole effective mass and scattering mechanism in strained $\mathrm{Ge}$ channel structures. Appl. Phys. Lett. 95, 122109 (2009).

159. Foronda, J., Morrison, C., Halpin, J. E., Rhead, S. D. \& Myronov, M. Weak antilocalization of high mobility holes in a strained Germanium quantum well heterostructure. J. Phys. Condens. Matter 27 022201 (2015).

160. Hassan, A. H. A. et al. Anisotropy in the hole mobility measured along the $[110]$ and $[1-10]$ orientations in a strained Ge quantum well. Appl. Phys. Lett. 104 $132108(2014)$

161. Morrison, C. et al. Observation of Rashba zero-field spin splitting in a strained germanium 2D hole gas. Appl. Phys. Lett. 105, 182401 (2014).

162. Failla, M., Myronov, M., Morrison, C., Leadley, D. R. \& Lloyd-Hughes, J. Narrow heavy-hole cyclotron resonances split by the cubic Rashba spin-orbit interaction in strained germanium quantum wells. Phys. Rev. B 92, 045303 (2015)

163. Shi, Q., Zudov, M. A., Morrison, C. \& Myronov, M Spinless composite fermions in an ultrahigh-quality strained Ge quantum well. Phys. Rev. B 91, 241303 (2015).

164. Morrison, C., Casteleiro, C., Leadley, D. R. \& Myronov, M. Complex quantum transport in modulation doped strained Ge quantum well heterostructure with a high mobility 2D hole gas. Appl. Phys. Lett. 109, 102103 (2016)

165. Holmes, S. N. et al. Spin-splitting in p-type Ge devices. J. Appl. Phys. 120, 085702 (2016).
166. Mironov, O. et al. Fractional quantum hall states in a Ge quantum well. Phys. Rev. Lett. 116, 176802 (2016).

167. Morrison, C. \& Myronov, M. Electronic transport anisotropy of 2D carriers in biaxial compressive strained germanium. Appl. Phys. Lett. 111, 192103 (2017).

168. Drichko, I. L. et al. Effective g factor of 2D holes in strained Ge quantum wells. J. Appl. Phys. 123 165703 (2018).

169. Berkutov, I. B., Andrievskii, V. V., Kolesnichenko, Y. A. \& Mironov, O. A. Quantum effects in a germanium quantum well with ultrahigh mobility of charge carrier. Low Temp. Phys. 45, 1202-1208 (2019).

170. Laroche, D. et al. Magneto-transport analysis of an ultra-low-density two-dimensional hole gas in an undoped strained $\mathrm{Ge} / \mathrm{SiGe}$ heterostructure. Appl. Phys. Lett. 108, 233504 (2016).

171. Lu, T. M. et al. Density-controlled quantum Hall ferromagnetic transition in a two-dimensional hole system. Sci. Rep. 7, 2468 (2017).

172. Lu, T. M. et al. Effective $g$ factor of low-density two-dimensional holes in a Ge quantum well. Appl. Phys. Lett. 111, 102108 (2017)

173. Hardy, W. J. et al. Single and double hole quantum dots in strained Ge/SiGe quantum wells. Nanotechnology 30 215202 (2019).

174. Failla, M. et al. Terahertz quantum Hall effect for spin-split heavy-hole gases in strained Ge quantum wells. New J. Phys. 18, 113036 (2016).

175. Gul, Y. et al. Quantum ballistic transport in strained epitaxial germanium. Appl. Phys. Lett. 111, 233512 (2017).

176. Mizokuchi, R., Maurand, R., Vigneau, F., Myronov, M. $\&$ De Franceschi, S. Ballistic one-dimensional holes with strong $g$-factor anisotropy in germanium. Nano Lett. 18, 4861-4865 (2018).

177. Gul, Y., Holmes, S. N., Myronov, M., Kumar, S. \& Pepper, M. Self-organised fractional quantisation in a hole quantum wire. J. Phys. Condens. Matter 30 , 09LT01 (2018)

178. Lauhon, L. J., Gudiksen, M. S., Wang, D. \& Lieber, C. M. Epitaxial core-shell and core-multishell nanowire heterostructures. Nature 420, 57-61 (2002).

179. Morales, A. M. \& Lieber, C. M. A laser ablation method for the synthesis of crystalline semiconductor nanowires. Science 279, 208-211 (1998)

180. Woodruff, J. H., Ratchford, J. B., Goldthorpe, I. A., McIntyre, P. C. \& Chidsey, C. E. D. Vertically oriented germanium nanowires grown from gold colloids on silicon substrates and subsequent gold removal. Nano Lett. 7, 1637-1642 (2007).

181. Dayeh, S. A. \& Picraux, S. T. Direct observation of nanoscale size effects in Ge semiconductor nanowire growth. Nano Lett. 10, 4032-4039 (2010).

182. Tian, B., Xie, P., Kempa, T. J., Bell, D. C. \& Lieber, C. M. Single-crystalline kinked semiconductor nanowire superstructures. Nat. Nanotechnol. 4, 824-829 (2009).

183. Goldthorpe, I. A., Marshall, A. F. \& McIntyre, P. C Inhibiting strain-induced surface roughening: dislocation-free $\mathrm{Ge} / \mathrm{Si}$ and $\mathrm{Ge} / \mathrm{SiGe}$ core-shell nanowires. Nano Lett. 9, 3715-3719 (2009).

184. Conesa-Boj, S. et al. Boosting hole mobility in coherently strained [110]-oriented Ge-Si core-shell nanowires. Nano Lett. 17, 2259-2264 (2017).

185. Dillen, D. C., Kim, K., Liu, E.-S. \& Tutuc, E. Radial modulation doping in core-shell nanowires. Nat. Nanotechnol. 9, 116-120 (2014).

186. Sistani, M. et al. Highly transparent contacts to the 1D hole gas in ultrascaled $\mathrm{Ge} / \mathrm{Si}$ core/shell nanowires. ACS Nano 13, 14145-14151 (2019).

187. Lu, W., Xiang, J., Timko, B. P., Wu, Y. \& Lieber, C. M. One-dimensional hole gas in germanium/silicon nanowire heterostructures. Proc. Natl Acad. Sci. USA 102, 10046-10051 (2005)

188. Kotekar-Patil, D., Nguyen, B.-M., Yoo, J., Dayeh, S. A. \& Frolov, S. M. Quasiballistic quantum transport through $\mathrm{Ge} / \mathrm{Si}$ core/shell nanowires. Nanotechnology 28, 385204 (2017)

189. Zhang, X., Jevasuwan, W., Sugimoto, Y. \& Fukata, N. Controlling catalyst-free formation and hole gas accumulation by fabricating $\mathrm{Si} / \mathrm{Ge}$ core-shell and $\mathrm{Si} / \mathrm{Ge} / \mathrm{Si}$ core-double shell nanowires. ACS Nano 13 13403-13412 (2019).

190. Tersoff, J. \& Tromp, R. M. Shape transition in growth of strained islands: spontaneous formation of quantum wires. Phys. Rev. Lett. 70, 2782-2785 (1993).

191. Mo, Y.-W., Savage, D. E., Swartzentruber, B. S. \& Lagally, M. G. Kinetic pathway in Stranski-Krastanov growth of Ge on $\mathrm{Si}(001)$. Phys. Rev. Lett. 65 1020-1023 (1990).
192. McKay, M. R., Venables, J. A. \& Drucker, J. Kinetically suppressed Ostwald ripening of $\mathrm{Ge} / \mathrm{Si}(100)$ hut clusters. Phys. Rev. Lett. 101, 216104 (2008).

193. Zhang, J. J. et al. Monolithic growth of ultrathin Ge nanowires on Si(001). Phys. Rev. Lett. 109, 085502 (2012).

194. Tersoff, J. \& LeGoues, F. K. Competing relaxation mechanisms in strained layers. Phys. Rev. Lett. 72, 3570-3573 (1994).

195. Watzinger, H., Glaser, M., Zhang, J. J., Daruka, I. \& Schäffler, F. Influence of composition and substrate miscut on the evolution of $\{105\}$-terminated in-plane $\mathrm{Si}_{1-x} \mathrm{Ge}_{x}$ quantum wires on $\mathrm{Si}(001)$. APL Mater. $\mathbf{2}$, 076102 (2014).

196. Brauns, M. et al. Highly tuneable hole quantum dots in Ge-Si core-shell nanowires. Appl. Phys. Lett. 109 143113 (2016).

197. Froning, F. N. M. et al. Single, double, and triple quantum dots in Ge/Si nanowires. Appl. Phys. Lett. 113, 073102 (2018)

198. Roddaro, S. et al. Spin states of holes in Ge/Si nanowire quantum dots. Phys. Rev. Lett. 101, 186802 (2008).

199. Brauns, M. et al. Anisotropic Pauli spin blockade in hole quantum dots. Phys. Rev. B 94, 041411 (2016).

200. Zarassi, A. et al. Magnetic field evolution of spin blockade in $\mathrm{Ge} / \mathrm{Si}$ nanowire double quantum dots. Phys. Rev. B 95, 155416 (2017).

201. Vukušic ${ }^{-}$, L. et al. Single-shot readout of hole spins in Ge. Nano Lett. 18, 7141-7145 (2018).

202. Vukušic`, L., Kukuc`ka, J., Watzinger, H. \& Katsaros, G. Fast hole tunneling times in germanium hut wires probed by single-shot reflectometry. Nano Lett. 17 5706-5710 (2017).

203. Hanson, R., Kouwenhoven, L. P., Petta, J. R., Tarucha, S. \& Vandersypen, L. M. K. Spins in few-electron quantum dots. Rev. Mod. Phys. 79, 1217-1265 (2007).

204. Lawrie, W. I. L. et al. Quantum dot arrays in silicon and germanium. Appl. Phys. Lett. 116, 080501 (2020).

205. Hofmann, A. et al. Assessing the potential of Ge/SiGe quantum dots as hosts for singlet-triplet qubits. Preprint at arXiv https://arxiv.org/abs/1910.05841 (2019).

206. Higginbotham, A. P. et al. Hole spin coherence in a Ge/Si heterostructure nanowire. Nano Lett. 14 , 3582-3586 (2014).

207. Vandersypen, L. M. K. et al. Interfacing spin qubits in quantum dots and donors - hot, dense, and coherent NPJ Quantum Inf. 3, 34 (2017).

208. Lawrie, W. I. L. et al. Spin relaxation benchmarks and individual qubit addressability for holes in quantum dots. Nano Lett. 20, 7237-7242 (2017).

209. Hutin, L. et al. in Proceedings of the 2018 48th European Solid-State Device Research Conference (ESSDERC) 12-17 (IEEE, 2018).

210. Froning, F. N. M. et al. Ultrafast hole spin qubit with gate-tunable spin-orbit switch. Preprint at $a r X i v$ http://arxiv.org/abs/2006.11175 (2020).

211. Knill, E. et al. Randomized benchmarking of quantum gates. Phys. Rev. A 77, 012307 (2008)

212. Bertrand, B. et al. Quantum manipulation of two-electron spin states in isolated double quantum dots. Phys. Rev. Lett. 115, 096801 (2015).

213. De Franceschi, S., Kouwenhoven, L., Schönenberger, C. $\S$ Wernsdorfer, W. Hybrid superconductor-quantum dot devices. Nat. Nanotechnol. 5, 703-711 (2010).

214. Lutchyn, R. M. et al. Majorana zero modes in superconductor-semiconductor heterostructures. Nat. Rev. Mater. 3, 52-68 (2018).

215. Clark, T. D., Prance, R. J. \& Grassie, A. D. C. Feasibility of hybrid Josephson field effect transistors. J. Appl. Phys. 51, 2736 (1980).

216. de Lange, G. et al. Realization of microwave quantum circuits using hybrid superconducting-semiconducting nanowire Josephson elements. Phys. Rev. Lett. 115, 127002 (2015).

217. Casparis, L. et al. Superconducting gatemon qubit based on a proximitized two-dimensional electron gas. Nat. Nanotechnol. 13, 915-919 (2018).

218. Sau, J. D. \& Sarma, S. D. Realizing a robust practical Majorana chain in a quantum-dot-superconductor linear array. Nat. Commun. 3, 964 (2012).

219. Ridderbos, J. et al. Multiple Andreev reflections and Shapiro steps in a Ge-Si nanowire Josephson junction. Phys. Rev. Mater. 3, 084803 (2019)

220. Ridderbos, J. et al. Hard superconducting gap and diffusion-induced superconductors in $\mathrm{Ge}-\mathrm{Si}$ nanowires. Nano Lett. 20, 122-130 (2020).

221. Hendrickx, N. W. et al. Ballistic supercurrent discretization and micrometer-long Josephson coupling in germanium. Phys. Rev. B 99, 075435 (2019). 
222. De Franceschi, S. et al. Andreev reflection in Si-engineered $\mathrm{Al} / \mathrm{InGaAs}$ hybrid junctions. Appl. Phys. Lett. 73, 3890-3892 (1998).

223. Krogstrup, P. et al. Epitaxy of semiconductorsuperconductor nanowires. Nat. Mater. 14 400-406 (2015)

224. Kral, S. et al. Abrupt Schottky junctions in $\mathrm{Al} / \mathrm{Ge}$ nanowire heterostructures. Nano Lett. 15, 4783-4787 (2015)

225. El Hajraoui, K. et al. In situ transmission electron microscopy analysis of aluminum-germanium nanowire solid-state reaction. Nano Lett. 19 , 2897-2904 (2019).

226. Franke, D. P., Clarke, J. S., Vandersypen, L. M. K. \& Veldhorst, M. Rent's rule and extensibility in quantum computing. Microprocess. Microsyst. 67, 1-7 (2019).

227. Taylor, J. M. et al. Fault-tolerant architecture for quantum computation using electrically controlled semiconductor spins. Nat. Phys. 1, 177-183 (2005).

228. Veldhorst, M., Eenink, H. G. J., Yang, C. H. \& Dzurak, A. S. Silicon CMOS architecture for a spin-based quantum computer. Nat. Commun. 8 , 1766 (2017).

229. Li, R. et al. A crossbar network for silicon quantum dot qubits. Sci. Adv. 4, eaar3960 (2018).

230. Hill, C. D. et al. A surface code quantum computer in silicon. Sci. Adv. 1, e1500707 (2015).

231. Nickerson, N. H., Li, Y. \& Benjamin, S. C. Topological quantum computing with a very noisy network and local error rates approaching one percent. Nat. Commun. 4, 1756 (2013).

232. Veldhorst, M. et al. A two-qubit logic gate in silicon. Nature 526, 410-414 (2015).

233. Watson, T. F. et al. A programmable two-qubit quantum processor in silicon. Nature 555, 633-637 (2018).

234. Zheng, G. et al. Rapid gate-based spin read-out in silicon using an on-chip resonator. Nat. Nanotechnol. 14, 742-746 (2019).

235. West, A. et al. Gate-based single-shot readout of spins in silicon. Nat. Nanotechnol. 14, 437-441 (2019).

236. Urdampilleta, M. et al. Gate-based high fidelity spin readout in a CMOS device. Nat. Nanotechnol. 14 737-741 (2019).

237. Crippa, A. et al. Gate-reflectometry dispersive readout and coherent control of a spin qubit in silicon Nat. Commun. 10, 2776 (2019).

238. Samkharadze, N. et al. Strong spin-photon coupling in silicon. Science 359, 1123-1127 (2018).
239. Mi, X. et al. A coherent spin-photon interface in silicon. Nature 555, 599-603 (2018)

240. Borjans, F., Croot, X. G., Mi, X., Gullans, M. J. \& Petta, J. R. Resonant microwave-mediated interactions between distant electron spins. Nature 577, 195-198 (2020).

241. Fowler, A. G., Mariantoni, M., Martinis, J. M. \& Cleland, A. N. Surface codes: Towards practical large-scale quantum computation. Phys. Rev. A 86 032324 (2012).

242. Itoh, K. M. \& Watanabe, H. Isotope engineering of silicon and diamond for quantum computing and sensing applications. MRS Commun. 4, 143-157 (2014).

243. Hu, X., Liu, Y.-x. \& Nori, F. Strong coupling of a spin qubit to a superconducting stripline cavity. Phys. Rev. B 86, 035314 (2012).

244. Sigrist, M. \& Ueda, K. Phenomenological theory of unconventional superconductivity. Rev. Mod. Phys. 63, 239-311 (1991)

245. Sau, J. D., Lutchyn, R. M., Tewari, S. \& Das Sarma, S. Generic new platform for topological quantum computation using semiconductor heterostructures. Phys. Rev. Lett. 104, 040502 (2010).

246. Alicea, J. Majorana fermions in a tunable semiconductor device. Phys. Rev. B 81, 125318 (2010).

247. Read, N. \& Green, D. Paired states of fermions in two dimensions with breaking of parity and time-reversal symmetries and the fractional quantum Hall effect. Phys. Rev. B 61, 10267-10297 (2000).

248. Nayak, C., Simon, S. H., Stern, A., Freedman, M. \& Das Sarma, S. Non-Abelian anyons and topological quantum computation. Rev. Mod. Phys. 80, 1083-1159 (2008)

249. Mao, L., Shi, J., Niu, Q. \& Zhang, C. Superconducting phase with a chiral $f$-wave pairing symmetry and Majorana fermions induced in a hole-doped semiconductor. Phys. Rev. Lett. 106, 157003 (2011).

250. Leijnse, M. \& Flensberg, K. Quantum information transfer between topological and spin qubit systems. Phys. Rev, Lett. 107, 210502 (2011).

251. Leijnse, M. \& Flensberg, K. Hybrid topological-spin qubit systems for two-qubit-spin gates. Phys. Rev. $B$ 86, 104511 (2012)

252. Hoffman, S., Schrade, C., Klinovaja, J. \& Loss, D. Universal quantum computation with hybrid spin-Majorana qubits. Phys. Rev. B 94, 045316 (2016).
253. Ranc 'ic`, M. J., Hoffman, S., Schrade, C., Klinovaja, J. $\&$ Loss, D. Entangling spins in double quantum dots and Majorana bound states. Phys. Rev. B 99, 165306 (2019).

254. Choi, M.-S., Bruder, C. \& Loss, D. Spin-dependent Josephson current through double quantum dots and measurement of entangled electron states. Phys. Rev. B 62, 13569-13572 (2000).

255. Leijnse, M. \& Flensberg, K. Coupling spin qubits via superconductors. Phys. Rev. Lett. 111, 060501 (2013).

256. Hassler, F., Catelani, G. \& Bluhm, H. Exchange interaction of two spin qubits mediated by a superconductor. Phys. Rev. B 92, 235401 (2015).

257. Wang, K. et al. Ultrafast operations of a hole spin qubit in Ge quantum dot. Preprint at $a r X i v$ http://arxiv.org/abs/2006.12340 (2020).

\section{Acknowledgements}

G.S., M.V. and F.A.Z. acknowledge financial support from the Netherlands Organization for Scientific Research (NWO). F.A.Z., D.L. and G.K. acknowledge funding from the European Union's Horizon 2020 research and innovation programme under grant agreement no. 862046. G.K. acknowledges funding from FP7 ERC Starting Grant 335497, FWF Y 715-N30 and FWF P-30207. S.D.F. acknowledges support from the European Union's Horizon 2020 research and innovation programme under grant agreement no. 81050 and from the Agence Nationale de la Recherche through the TOPONANO and OSPIN projects. J.-J.Z. acknowledges support from the National Key R\&D Program of China (grant no. 2016YFA0301701) and Strategic Priority Research Program of the Chinese Academy of Sciences (grant no. XDB30000000). D.L. and C.K. acknowledge the Swiss National Science Foundation and NCCR OSIT.

\section{Author contributions}

All authors contributed to the writing of the manuscript.

\section{Competing interests}

The authors declare no competing interests.

\section{Publisher's note}

Springer Nature remains neutral with regard to jurisdictional claims in published maps and institutional affiliations.

(C) Springer Nature Limited 2020 